\title{
Moving from customer lifetime value to customer equity
}

\author{
Xavier Drèze • André Bonfrer
}

Received: 25 September 2008 / Accepted: 22 June 2009 /Published online: 31 July 2009

(C) The Author(s) 2009. This article is published with open access at Springerlink.com

\begin{abstract}
We study the consequence of moving from Customer Lifetime Value maximization to Customer Equity maximization. Customer equity has traditionally been seen as the discounted sum of the lifetime earnings from all current and future customers and thus it has been largely assumed that maximizing customer lifetime value would lead to maximum customer equity. We show that the transition from $C L V$ to $C E$ is not that straightforward. Although the $C L V$ model is appropriate for managing a single non-replaceable customer, the application of a $C L V$ model to the acquisition and valuation of customers as an ongoing concern for the firm leads to sub-optimal customer relationship management and acquisition strategies. This leads the firm following a $C L V$ maximization approach to have a smaller and less profitable customer base than one that follows a $C E$ maximization strategy.
\end{abstract}

Keywords Customer equity - Database marketing - Customer lifetime value · Internet marketing · Direct marketing · Customer acquisition · Customer retention · Customer relationship management

JEL Classification $\mathrm{M} 31 \cdot \mathrm{C} 51 \cdot \mathrm{Q} 20$

This research was funded in part by the Wharton-SMU research center, Singapore Management University and in part by a WeBI-Mac Center Grant.

Electronic supplementary material The online version of this article (doi:10.1007/s11129-009-9067-y) contains supplementary material, which is available to authorized users.

X. Drèze $(\bowtie)$

UCLA Anderson School of Management, 110 Westwood Plaza, Los Angeles, CA 90095-1481, USA

e-mail: xavier.dreze@anderson.ucla.edu

A. Bonfrer

Lee Kong Chian School of Business, Singapore Management University, Singapore, Singapore 


\section{Introduction}

In an era when Customer Relationship Management (CRM) is widely espoused, many researchers extol the virtues of Customer lifetime value $(C L V)$ as the best metric to use to select customers and optimize marketing actions (Farris et al 2006; Fader et al. 2005; Reinartz and Kumar 2003). A customer's lifetime value is the net present value of all profits derived from that customer. It is constructed using core metrics such as customer retention rate, revenue per customer, and discount rate of money. Berger and Nasr (1998) discuss the concept in detail and provide many different ways to compute it depending on the situation the firm is facing. Proponents of $C L V$ argue that it should be used for customer acquisition (CLV is the upper bound of what one should be willing to spend acquiring a customer lest one wants to lose money-Farris et al. 2006; Berger and Nasr 1998), customer selection (one should focus on customer with high $C L V$-Venkatesan and Kumar 2004), and resource allocation (marketing resources should be allocated so as to maximize $C L V$-Reinartz et al. 2005; Venkatesan and Kumar 2004).

Along with this renewed interest in CLV, there has been a move towards using Customer Equity $(C E)$ as a marketing metric both for assessing the return of marketing actions and to value firms as a whole. The metric, proposed by Blattberg and Deighton (1996) and defined by Rust et al. (2004) as "the total of the discounted lifetime values summed over all the firm's current and potential customers," seeks to assess the value of not only a firm's current customer base, but also its potential or future customer base as well. This long term view seems appropriate since a firm's actions at any given time do not only affect its customer base at that point in time, but they also affect its ability to recruit and retain customers in subsequent time periods. Customer Equity has been endorsed by many researchers. For instance, Berger et al. (2002) make $C E$ the foundation on which they build their framework for managing customers as assets; Bayon et al. (2002) make a case that $C E$ is the best basis for customer analysis; Gupta et al. (2004) use $C E$ as a basis for valuing firms. Villanueva et al. (2008) use $C E$ to measure the impact of different acquisition methods on the long term value of the firm.

There is a direct link between $C L V$ and $C E$ : customer equity is the sum of the $C L V$ of all current and future customers. When building $C E$ as an aggregation of $C L V \mathrm{~s}$, researchers have taken for granted that the precepts coming out of a $C L V$ analysis still apply when looking at customer equity (e.g., $C L V$ is a limit to acquisition spending; maximizing $C L V$ is equivalent to maximizing $C E$ ). The distinction between $C L V$ and $C E$ is confounded by the fact that some researchers (e.g., Berger and Bechwatti 2002) use the terms interchangeably. There is no doubt however that the two concepts are distinct; $C L V$ computes the value of a customer to the firm while $C E$ measures the value of all present and future customers given a firm's marketing actions (e.g., acquisition policy, marketing mix). There is no doubt that when valuing a firm (Gupta et al. 2004) one should use $C E$ as a metric rather than $C L V$. But, when optimizing marketing decisions, are $C L V$ and $C E$ interchangeable? Since $C E$ is a sum of $C L V$, are we maximizing $C E$ when we maximize $C L V$ ? The theoretical development we conduct in this paper shows that the answer to these two questions is a resounding No. Maximizing $C L V$ is sub-optimal from a $C E$ perspective, it leads to the wrong marketing actions and the wrong acquisition 
policy. We find that firms that optimize $C L V$ generate lower profits and retain fewer customers than firms that optimize CE.To show why $C L V$ is sub-optimal, we start with a discussion of customers as a resource to the firm (Section 2.1) and show that a firm should aim to maximize the long-term value of this resource. Based on this discussion, we derive a general functional form for a firm's Customer Equity in Section 2.3. In Sections 3.1 and 4, we derive the first order condition that must be satisfied to maximize $C E$ and perform comparative statics to show how marketing actions should be adapted when control variables (e.g., retention rate, acquisition effectiveness) change. We show how maximizing $C E$ is different from maximizing $C L V$ such that maximizing $C L V$ is sub-optimal from a customer equity standpoint. Then, in Section 5, we discuss the impact of a long term focus on acquisition spending. We show that firms that aim to maximize $C E$ will spend less on acquisition than a firm that aims to maximize $C L V$; however, it will retain its customers longer and generate larger total profits. In Section 6, we show our model to be robust to both observed and un-observed heterogeneity. We conclude the paper in Section 7.

\section{A customer equity model}

For modeling purposes, and in the spirit of Berger et al. (2002), we consider the case of a firm that is trying to generate revenues by contacting members of its database. Customers are recruited through an acquisition policy and are contacted at fixed intervals. The problem for the firm is to set its marketing policy, defined here as the contact periodicity and the amount of money spent on acquisition, that will maximize its expected revenues, taking into account the various costs it faces (acquisition, communication) and the reaction of customers to the firm's policy (defection, purchase).

When developing our model of customer equity, we pay particular attention to two processes. First, we seek to capture the customer acquisition/retention process in a meaningful way. Second, we seek to capture the impact of the firm's marketing actions on customer retention and expenditure.

\subsection{Customer flow: acquisition and retention process}

Fundamentally, managing a database of customer names is a dynamic problem that hinges on balancing the cultivation of surplus from the firm's customers with the retention of customers for future rent extraction. The problem of extracting the highest possible profits from a database of customer names is similar to the challenges encountered by researchers studying the management of natural resources. Economists (Sweeny 1992) consider three types of resources: depletable, renewable, and expendable. The distinction is made based on the time scale of the replenishment process. Depletable resources, such as crude oil reserves, are those for which the replenishment process is so slow that one can model them as being available once and only once and with the recognition that spent resources cannot be replaced. Renewable resources, such as the stock of fish in a lake or pond, adjust more rapidly so that they renew within the time horizon studied. However, any 
action in a given time period that alters the stock of the resource will have an impact on the stock available in subsequent periods. Finally, expendable resources, such as solar energy, renew themselves at such a speed that their use in one period has little or no impact on subsequent periods.

Depending on the type of resource a firm is managing, it faces different objectives. In the case of a depletable resource, the firm is interested in the optimal use of the resource in its path to depletion. With a renewable resource, the firm is interested in long-term equilibrium strategies. Faced with an expendable resource, the firm is interested in its best short-term allocation.

When looking at its customer database, a firm can treat its customers as any of these three resource types depending on the lens it uses for the problem. In the cohort view (the basis for $C L V$ ), customers are seen as a depletable resource. New cohorts of customers are recruited over time, but each cohort will eventually become extinct (Wang and Spiegel 1994). Attrition is measured by the difference in cohort size from 1 year to another. In an email-SPAM environment, where consumers cannot prevent the firm from sending them emails, and where new email names can readily be found by "scouring" the Internet, the firm views consumers as an expendable resource and thus can take a short-term approach to profit maximization.

Customer equity takes a long term approach to valuing and optimizing a firm's customer base. For a firm with a long-term horizon, a depletable resource view of customers is not viable as it implies that the firm will lose all its customers at some point. Thus a customer acquisition process must be in place for the firm to survive. Further, if the firm observes defection and incurs acquisition costs to replace lost customers, then it cannot treat its customers as expendable. Hence, we argue that the appropriate way to look at the profit maximization problem is to view customers as a renewable resource. This view is the basis for our formulation and analysis of a firm's customer equity. Further, if the firm views customers as a renewable resource, then it must evaluate its policies in terms of the long-term equilibrium they generate. This is the approach we take in this paper.

\subsection{The impact of marketing actions on customer retention and expenditure}

One cannot capture in a simple model the impact of all possible marketing actions (pricing decisions, package design, advertising content...). Hence, as in Venkatesan and Kumar (2004), we concentrate on optimizing the frequency with which a firm should contact its customer base. It is a broadly applicable problem that is meaningful for many applications, not only for the timing of mail or email communications, but also the frequency of calls by direct sales force or the intensity of broadcast advertising. It also possesses some interesting characteristics that illustrate typical tensions among marketing levers in terms of spending on retention versus cultivation efforts. For instance, the effect of the periodicity of communication on attrition is not so straightforward. To see why, one can conduct the following thought experiment: let us consider the two extremes in contact periodicity. At one extreme, a firm might contact its clients so often that the relationship becomes too onerous for the clients to maintain and thus they sever their links to the company, rendering their names worthless. (Evidence of this phenomenon can be seen in the emergence of the 'Do Not Call Registry' in 2003 as well as the Anti-Spamming Act 
of 2001.) At the other extreme, the firm never contacts its customers and, although the names have a potential value, this value is never realized. Thus, periodicity affects the value of names in two ways. On the one hand, more frequent contact leads to more opportunities to earn money. On the other hand, more frequent contact provides customers with more opportunities to defect. The latter can quickly lead to high long-term attrition. Imagine the case of a company that has a retention rate of $97 \%$ from one campaign to another. This might at first glance seem like outstanding loyalty. However, if the firm were to contact its database every week then it would lose $80 \%$ of its current customers within a year! Clearly, there must be an intermediate situation where one maximizes the realized value from a name by optimally trading off the extraction of value in the short-term against the loss of future value due to customer defection.

\subsection{Customer equity}

Our customer equity model is in essence a version of the model developed by Gupta et al. (2004) that has been modified to directly take into account the requirement described in the preceding two sections. In particular, we make customer retention and customer spending a function of the time elapsed between communication $(\tau)$. We also explicitly develop a consumer production function $\left(S_{i}(\tau)\right)$ that characterizes how the customer base evolves from one period to another as a function of acquisition and retention.

Because the time elapsed between each communication is a control variable, we must be careful when discounting revenues and expenses. To apply the correct discount rate to expenses and revenues, one must pinpoint when each occurs. Consistent with Venkatesan and Kumar (2004), we assume that acquisition expenses are an ongoing expenditure that the firm recognizes on a periodic basis (e.g., monthly) while communications expenses and revenues are recognized at the time of the communication. This assumption is consistent with our observations of practice, where the acquisition costs are decoupled from the marketing activities once the names are acquired. This is also a recommendation of Blattberg and Deighton (1996), highlighting the different roles of acquisition and retention efforts.

Based on these assumptions, Customer Equity can be generally defined as:

$$
C E(\tau)=\sum_{i=0}^{\infty} e^{-i r t}\left(R_{i}(\tau) S_{i}(\tau)-F C_{i}\right)-\sum_{j=0}^{\infty} e^{-j r} A Q j .
$$

Where:

$\begin{array}{ll}\mathrm{i} & \text { is an index of communications } \\ \mathrm{j} & \text { is an index of time periods } \\ \mathrm{e}^{-\mathrm{r}} & \text { is the per period discount rate } \\ \tau & \text { is the periodicity of contact }\end{array}$

\footnotetext{
${ }^{1} N P V$ calculations traditionally uses $1 /(1+d)$, where $d$ is the discount rate of money, as the discount factor. To make our derivations simpler, we use $e^{-r}$ instead. The two formulations are equivalent if we set $r=\ln (1+d)$.
} 
$R_{i}(\tau)$ is the expected profit (revenues net of cost of goods sold and variable communication costs) per customer for communication ${ }_{i}$

$S_{i}(\tau)$ is the number of people in the database when communication $i$ was sent $\mathrm{FC}_{\mathrm{i}} \quad$ is the fixed cost associated with communication ${ }_{i}$

$\mathrm{AQ}_{\mathrm{j}} \quad$ is the acquisition costs incurred in time period $j$.

Further, we define the customer profit $\left(R_{i}(\tau)\right)$ and production $\left(S_{i}(\tau)\right)$ functions as:

$$
\begin{gathered}
R_{i}(\tau)=\left(A_{i}(\tau)-V C_{i}\right), \\
S_{i}(\tau)=S_{i-1}(\tau) P_{i}(\tau)+g_{i}(\tau) .
\end{gathered}
$$

Where:

$A_{i}(\tau)$ is the expected per-customer gross profit for communication $i$

$V C_{\mathrm{i}}$ is the variable cost of sending communication $i$

$S_{0}(\tau)=0$

$P_{i}(\tau) \quad$ is the retention rate for communication $i$

$g_{i}(\tau) \quad$ is the number of names acquired between campaigns $i-1$ and $i$.

The model laid out above is a general case that is applicable to many situations. However, we make the following simplifications in order to make the maximization problem more tractable. First, we assume that the acquisition efforts are constant over time and produce a steady stream of names (i.e., $\mathrm{g}_{\mathrm{i}}(\tau)=\tau$.g, $\mathrm{AQ}_{\mathrm{j}}=\mathrm{AQ}(\mathrm{g})$ ). There is no free production of names and the cost of acquisition increases with the size of the name stream such that: $A Q(0)=0, \partial A Q(g) / \partial g>0$. Second, we assume that the fixed and variable communications costs are constant across communications (i.e., $V C_{i}=V C, F C_{i}=F C$ ). Third, we assume that the communications are identical in nature, if not in actual content, and that customers' reaction to the communications depends only on their frequency such that: $A_{i}(\tau)=A(\tau)$ and $P_{i}(\tau)=P(\tau)$. Further, we assume that $\mathrm{A}(\tau)$ is monotonically increasing in $\tau$, with A $(0)=0$ and $\mathrm{A}(\infty)$ finite (i.e., the more time the firm has to come up with a new offer, the more attractive it can make it - up to a point), and that $P(\tau)$ is inverted-U shaped (i.e., retention is lowest when the firm sends incessant messages or when it never contacts its customers and there is a unique optimal communication periodicity for retention purposes) and dependent on $A(\tau)$ such that $\partial P(\tau) / \partial A(\tau)>0$ (i.e., the better the offer, the higher the retention rate).

We assume, for now, that customers are treated by the firm as homogenous entities; i.e., there is no observed heterogeneity the firm can use to derive different $\tau$ for different individual. This is consistent with the recommendation of a number of CLV researchers (Zeithaml et al. 2001; Berger et al. 2002; Libai et al. 2002; Hwang et al. 2004) who talk about generating a set of rules for groups (or segments) of customers. It is also an approach that is widely followed by practitioners, customers are often segmented into fairly homogenous subgroups and then each subgroup is treated independently. With this in mind, we would argue that each customer segment has its own $C E$ with its own acquisition and retention process; Eq. (1) can then be applied to each of these segments. We will return to the issue of heterogeneity in Section 6. 
We defer to the technical appendix for a more complete justification of our assumptions. We also discuss the robustness of the major findings to the relaxation of these assumptions later in this paper when appropriate. Building on these assumptions, we can rewrite the customer equity equations as follows:

$$
\begin{gathered}
C E(\tau)=\sum_{i=0}^{\infty} e^{-i r \tau}\left(R(\tau) S_{i}(\tau)-F C\right)-\sum_{j=0}^{\infty} e^{-j r} A Q(g) \\
S_{i}(\tau)=S_{i-1}(\tau) P(\tau)+\tau . g \\
R(\tau)=(A(\tau)-V C) .
\end{gathered}
$$

We show (Lemma 1) that, given (5), in the long run, the database reaches an equilibrium size, regardless of the number of names the firm is endowed with at time $0\left(S_{0}\right)$.

Lemma 1 For any given constant marketing actions there exists a steady state such that the database is constant in size. The steady state size is $\bar{S}=\frac{\tau . g}{1-P(\tau)}$.

Proof See Appendix A.1.

The intuition behind Lemma 1 is straightforward given the database production function (5). For any given $\tau$ the firm acquires a constant number of new names $(\tau . g)$ between every communication, but it loses names in proportion $(P(\tau))$ to its database size. Consequently, the database will be at equilibrium when the firm, between one communication and the next, acquires as many names as it loses due to the communication.

This is analogous to Little's Law, which states that an inventory reaches a steady state size, $L$, which is a product of the arrival rate of new stock $(\lambda)$ and the expected time spent by any single unit of stock in the inventory $(W)$ or $L=\lambda W$ (Little 1961). When Little's Law is applied to an inventory of customer names we have $L=\bar{S}$ the expected number of units in the system, $\lambda-\tau . g$ the arrival rate of new units, and $W=1 /(1-P(\tau))$ the expected time spent by a unit in the system. Little's Law yields a finite inventory size, as long as $\lambda$ and $W$ are both finite and stationary. This law has been the subject of numerous papers and has been shown to hold under very general assumptions. This means that Lemma 1 will hold (in expected value) for any stochastic acquisition and retention process as long as they are stationary in the long run, that $\tau$ and $g$ are finite, and $P(\tau)<1$. The appeal of Little's Law applied to the $\mathrm{CE}$ problem is its robustness. This relationship holds even if:

- there is seasonality in retention or acquisition (aggregated to the year level, these processes become stationary-e.g., $g=E$ [Yearly acquisition rate]),

- the firm improves its ability to attract new customers (as long as $g=\lim _{t \rightarrow \infty} g(t)$ is finite, where $t$ is the length of time the firm has been in business),

- there is heterogeneity in customer retention (see Section 6),

- customer retention increases-possibly due to habit formation or inertia-as customers stay in the database longer (provided that $\lim P(\tau, t)<1$, where $t$ is the length of time during which a customer has been active). 
Lemma 1 yields some interesting properties for the $C E$ model. First, it allows managers to ex ante predict the long-term database size and $C E$. All they need to know is their long-term attrition rate and acquisition effectiveness. This is particularly important when valuating young companies (e.g., start-ups) as it provides a monitoring tool and a base for predicting long-term value. Second, it allows us to further simplify the formulation of $C E$. Indeed, we can write the steady state $C E$ :

$$
C E(\tau)=\sum_{i=0}^{\infty} e^{-i r \tau}(R(\tau) \bar{S}(\tau)-F C)-\sum_{j=0}^{\infty} e^{-j r} A Q(g)
$$

or, taking the limit of sums:

$$
C E(\tau)=(R(\tau) \bar{S}(\tau)-F C) \frac{e^{r \tau}}{e^{r \tau}-1}-A Q(g) \frac{e^{r}}{e^{r}-1} .
$$

Third, the elements of (7) are quite easy to compute for database marketers. Having a database of names they track, they can measure $\bar{S}$ (it is the number of live names in the database), they can compute $R$ as the average per-customer profit per campaign, the various costs can be obtained from their accounting department (as is $r$ ). Finally, they can set $\tau$ to maximize $C E(\tau)$ as discussed in Proposition 2, below.

Fourth, the optimal marketing actions and the resulting steady state database size is a function of the rate of the acquisition stream, not its cost. We formalize this point in Lemma 2.

Lemma 2 Given an acquisition stream, the marketing actions that maximize CE depend on the rate of acquisition, but are separable from the cost of acquisition.

Proof The proof is straightforward. One only needs to recognize that $C E$ can be split in two terms. The first one depends on $g$ and $\tau$, the second one depends on $g$ only. That is:

$$
C E(\tau)=\underbrace{(R(\tau) \bar{S}(\tau)-F C) \frac{e^{r \tau}}{e^{r \tau}-1}}_{f(\tau, g)}-\underbrace{A Q(g) \frac{e^{r}}{e^{r}-1}}_{k(g)} .
$$

Further, to maximize $C E$ with respect to $\tau$ one computes $\frac{\partial C E(\tau)}{\partial \tau}$ such that:

$$
\frac{\partial C E(\tau)}{\partial \tau}=\frac{\partial}{\partial \tau} f(\tau, g)-\frac{\partial}{\partial \tau} k(g)=\frac{\partial}{\partial \tau} f(\tau, g)=0 .
$$

The essence of the proof stems from the observation that the acquisition expenditures precede and are separable from the frequency of contact or the message content. Once names have entered the database, it is the marketer's responsibility to maximize the expected profits extracted from those names, treating the cost of acquisition as a sunk cost. That is, when optimizing the contact strategy, the firm only needs to know how many new names are acquired every month, not how much these names cost. Everything else being constant, two firms having the same acquisition rate, but different acquisition costs will have identical intercommunication intervals (but different overall profitability). 
Lemma 2 belies the belief that one should be more careful with names that were expensive to acquire than with names that were acquired at low cost. This does not imply, however, that acquisition costs are irrelevant. The longterm profitability of the firm relies on the revenues generated from the database being larger than the acquisition costs. Further, acquisition costs are likely to be positively correlated with the quality of the names acquired (where better quality is defined by either higher retention or higher revenue per customer). Nevertheless, the optimal marketing activity are a function of the acquisition rate $(g)$ and can be set without knowledge of the cost associated with this acquisition rate $(A Q(g))$.

The importance of Lemma 2 is that it allows us to split customer equity in two parts: the value of the customer database and the cost of replenishing the database to compensate attrition. We can thus study the optimization problem in two stages. First, solve (Section 3.1) the problem of maximizing the database value given a predetermined acquisition stream (i.e., find $\tau^{\uparrow *} \mid g$ ). Second, optimize (Section 5.1) the acquisition spending given the characterization of $\tau^{*}$. Further, this Lemma formalizes Blattberg and Deighton's (1996) suggestion that, when maximizing customer equity, the "acquisition" and the "customer equity" management tasks are very different, and should be treated separately.

\section{Customer equity and customer lifetime value}

We now turn to the profit maximization problem given an acquisition stream of names $(g)$. As we have shown in the previous section, if the acquisition expenditures are independent of $\tau$, we can ignore them when optimizing the communication strategy. The problem to the firm then becomes:

$$
\max _{\tau>0} V_{c e}(\tau)=(R(\tau) \bar{S}(\tau)-F C) \frac{e^{r \tau}}{e^{r \tau}-1}
$$

In this equation $V$ (the database value part of $C E$ ) represents the net present value of all future expected profits and the subscript ce indicates that we take a customer equity approach, as opposed to the customer lifetime value approach that we will formulate shortly (using $c l v$ as a subscript). We show in Proposition 1 that maximizing the $C L V$ leads to different solutions than maximizing $V_{c e}$ such that maximizing the $C L V$ is sub-optimal with regard to long-term customer equity maximization.

Proposition 1 CLV maximization is sub-optimal with regard to the long-term profitability of the firm.

Proof See Appendix A.2.

The intuition behind the proof is that when computing a $C L V$ one accounts for the fact that, due to attrition, customers have a decreasing probability of being active as time 
passes. Given our notation, one would write the $C L V$ of an individual customer as:

$$
C L V(\tau)=\sum_{i=0}^{\infty} e^{-i r \tau} P(\tau)^{i}\left(R(\tau)-\frac{F C}{\bar{S}}\right)=\left(R(\tau)-\frac{F C}{\bar{S}}\right) \frac{e^{r \tau}}{e^{r \tau}-P(\tau)}
$$

Thus, we can restate the database value (8) in terms of the customer lifetime value as:

$$
V_{c e}(\tau)=C L V(\tau) \bar{S} \frac{e^{r \tau}-P(\tau)}{e^{r \tau}-1}
$$

In this equation, the multiplier $\frac{e^{r \tau}-P(\tau)}{e^{r \tau}-1}>1$ accounts for the fact that customers are renewable and lost customers are replaced. The lower the retention rate, the higher the multiplier. Further, the multiplier increases as the discount rate $(r)$ or the communication interval $(\tau)$ decreases

Following Eq. (10), the database value is equal to the $C L V$ multiplied by a correction factor. Maximizing the $C L V$ will thus lead to the maximum customer equity if and only if, at the maximum $C L V$, the derivative of the multiplier with respect to $\tau$ is equal to 0 . We show in Appendix A. 2 that this can only occur if $\tau^{*}=0$. But, as we will show in the next section, $\tau^{*}$ is always strictly greater than zero. Thus, the marketing actions that maximize the $C L V$ do not also maximize the long term $C E$ of the firm. Therefore, CLV maximization is sub-optimal for the firm!

One should note that when computing the $C L V(9)$ we allocated a portion of the campaign costs $(F C)$ to each name. This allocation does not affect the substance of our findings. Indeed, if we were to ignore the fixed costs at the name level, we would compute the $C L V$ as:

$$
C L V(\tau)=R(\tau) \frac{e^{r \tau}}{e^{r \tau}-P(\tau)} .
$$

Thus, we would have:

$$
V_{c e}=[C L V(\tau) \bar{S}(\tau)] \frac{e^{r \tau}-P(\tau)}{e^{r \tau}-1}-F C \frac{e^{r \tau}}{e^{r \tau}-1} .
$$

The value of the database as a whole is the value of its names minus the costs associated with extracting profits from these names. In valuating each name we find the same multiplier $\frac{e^{r \tau}-P(\tau)}{e^{r \tau}-1}$ as we had when we incorporated the costs directly in the name value.

\subsection{Finding the optimal periodicity $\left(\tau^{*}\right)$}

In order to optimize the firm's marketing actions for any given acquisition strategy, we calculate the first-order condition for optimality by differentiating (8) with respect to $\tau$. Without further specifying the general functions that constitute the database value, it is not possible to generate an explicit closed-form solution for optimal $\tau$. However, we can make some inferences using comparative static tools. 
We start by describing the first-order condition, expressed as a function of the elasticities of the retention and profit functions with respect to changes in the intercommunication interval. Then, we study how the first-order condition changes as a result of changes in retention, profit, acquisition, and discount.

Proposition 2 The first-order condition for a firm that seeks to maximize its database value by optimizing the inter-communication interval is

$$
\eta_{R}+\eta_{\bar{S}}+\eta_{D} \cdot G M=0
$$

Where:

$$
\begin{aligned}
& \eta_{R}=\frac{\partial R(\tau)}{\partial \tau} \frac{\tau}{R(\tau)}=\frac{\partial A(\tau)}{\partial \tau} \frac{\tau}{A(\tau)-V C} \text { is the elasticity of } R(\tau) \text { with respect to } \tau, \\
& \eta_{\bar{S}}=1+\frac{\partial P(\tau)}{\partial \tau} \frac{\tau}{P(\tau)} \text { is the elasticity of } \bar{S} \text { with respect to } \tau, \\
& \eta_{D}=\frac{-r \tau}{e^{r \tau}-1} \text { is the elasticity of the discount multiplier }\left(D(\tau)=\frac{e^{r \tau}}{e^{r \tau}-1}\right) \text { with; respect to } \tau, \\
& G M=\frac{R(\tau) \cdot \bar{S}(\tau)-F C}{R(\tau) \cdot \bar{S}(\tau)} \text { is the gross margin yielded by each communication. }
\end{aligned}
$$

\section{Proof See Appendix B.1.}

The proof of Proposition 2 is an algebraic exercise that leads to a simple expression of the first-order condition: a linear combination of elasticities. The optimal inter-communication interval $\left(\tau^{*}\right)$ is found when the sum of elasticities is equal to 0 . When the sum is positive, the firm would increase its value by increasing $\tau$. When the sum is negative, the firm would be better off decreasing its $\tau$. As we show in the Technical Appendix, if there exists a $\tau$ such that $V_{c e}(\tau)$ is positive, then there exists a unique $\tau^{*}$ that is finite and strictly positive. This is not a restrictive condition, as it only assumes that it is possible for the firm to make some profits. If it were not the case, the firm will never be profitable and the search for an optimal $\tau$ becomes meaningless. Further, if $P(\tau)$ is not too convex then there are no local maxima and thus the maximum is unique. This, again, is not restrictive, as $P(\tau)$ will typically be concave over the domain of interest.

\section{The impact of retention rate, discounts, revenues, and acquisition rates on $C E$}

Our framework for the valuation of customer equity relies on several metrics commonly used to characterize a customer database: retention rate, acquisition rate, customer margins (revenues and costs), and discount rates. When any of these change, the marketing action $(\tau)$ must change to reach the new maximum customer equity. Since we endogenized the marketing action, we can now answer the question: how do these levers of customer value impact both the optimal marketing actions $(\tau *)$ and customer equity $\left(C E^{*}\right)$ ? To answer this question, we perform a comparative static analysis on 
the $C E$ problem. Furthermore, we make some generalization about how these levers impact customer equity.

\subsection{Change in retention rate $(P(\tau))$}

A change in the retention rate response function can come about in two different ways. First, one might see an intercept or level shift that increases or decreases $P(\tau)$ overall without changing the sensitivity of $P(\tau)$ to changes in $\tau$ (i.e., the gradient $\frac{\partial P(\tau)}{\partial \tau}$ is unaffected). Second, one might see a change in the sensitivity of $P(\tau)$ to changes in $\tau$ (i.e., a change of $\frac{\partial P(\tau)}{\partial \tau}$ at $\tau^{*}$ while $P\left(\tau^{*}\right)$ is constant). One might, of course, observe a combination of these two changes. In such a case, the total impact of the changes will be the sum of the changes due to the level-shift and the sensitivity-shift. The combined impact of both changes is given in the following proposition:

Proposition $3_{\mathbf{a}}$ An increase in retention sensitivity $\left(\frac{\partial P(\tau)}{\partial \tau}\right)$ leads to an increase in $\tau^{*}$. A level-shift increase in retention $(P(\tau))$ leads to an increase in $V_{c e}$. It also leads to an increase in $\tau^{*}$ when $\tau^{*}$ is small, and a decrease in $\tau^{*}$ when it is large. The cut-off level is: $\tau^{*}: \eta_{\bar{S}\left(\tau^{*}\right)}>\left|\eta_{D\left(\tau^{*}\right)}\right| \frac{F C}{R\left(\tau^{*}\right) \cdot \bar{S}\left(\tau^{*}\right)}+1$.

\section{Proof See Appendix B.2.}

The retention probability affects the $F O C$ through both $\eta_{\bar{S}}$ and $G M$. Thus, when looking at changes in $P(\tau)$ we need to consider the combined impact of both changes. In the case of a change in $\frac{\partial P(\tau)}{\partial \tau}$, the situation is straightforward, as $G M$ is unaffected and thus the increase in $\frac{\partial P(\tau)}{\partial \tau}$ leads to an increase in the $F O C$ through $\eta_{\bar{S}}$. Hence, the firm would react by increasing its $\tau$. In other words, an increase in $\frac{\partial P(\tau)}{\partial \tau}$ means that the firm has more to gain by waiting a little longer between communications, and since the system was at equilibrium before, it now leans in favor of a larger $\tau^{*}$.

In terms of database value, $V_{c e}$ is not directly affected by changes in $\frac{\partial P(\tau)}{\partial \tau}$ and thus, strictly speaking, a change in $\frac{\partial P(\tau)}{\partial \tau}$ will not affect the database value. However, a change in $\frac{\partial P(\tau)}{\partial \tau}$ cannot reasonably arise without some change in $P(\tau)$. And thus, $V_{c e}$ will be affected through the change in $P(\tau)$. It is straightforward to show that a levelshift increase in $P(\tau)$ increases $V_{c e}$. The envelope theorem tells us that although $\tau^{*}$ is affected by changes in $P$, when looking at the net impact on $V_{c e}$, we can ignore the (small) impact of changes in $P$ on $\tau^{*}$, and simply look at the sign of $\frac{\partial V_{C E}}{\partial P}$. Here, we have $\frac{\partial V_{C E}}{\partial P}>0$ and thus $V_{c e}$ is increasing in $P$.

In the case of a level-shift, the situation is made complex in that $G M$ always increases when $P(\tau)$ increases (which leads to a decrease in the $F O C$ as $\eta_{\mathrm{D}}$ is negative), but the effect on $\eta_{\bar{S}}$ depends on $\frac{\partial P(\tau)}{\partial \tau}$. If we assume that $P(\tau)$ is inverted$\mathrm{U}$ shaped (increasing at a decreasing rate for small $\tau-\frac{\partial V_{C E}}{\partial P}>0, \frac{\partial^{2} P(\tau)}{\partial \tau^{2}}<0, \forall \tau<\tau_{p}-$ then decreasing after some threshold- $\frac{\partial P(\tau)}{\partial \tau}<0, \forall \tau<\tau_{p}$ ) then we find that for small $\tau$ an intercept-shift increase in $P(\tau)$ leads to an increase in $\tau^{*}$, and for large $\tau$ it leads to a decrease in $\tau^{*}$. The difference in behavior comes from the fact that when $\tau$ is small, an increase in $P(\tau)$ has a large impact on the database size and spurs the company to seek an even larger database while, when $\tau$ is large, an increase in retention allows the firm to harvest the database to a greater extent. 


\subsection{Change in expected profit per contact $(R(\tau))$}

We study the impact of costs in the next section and focus here solely on $\mathrm{A}(\tau)$. Similar to $P(\tau), \mathrm{A}(\tau)$ can be changed through an intercept-shift or through a change in sensitivity to $\tau$. The situation is, however, a bit more complex for $\mathrm{A}(\tau)$ than for $P(\tau)$ because $P(\tau)$ depends on $\mathrm{A}(\tau)$. In other words, defection depends in part on the content that is sent to customers. Thus, we must account not only for the direct effect of a change in $\mathrm{A}(\tau)$ on the $F O C$, but also for indirect effects through changes in $P(\tau)$.

Proposition $\mathbf{3}_{\mathbf{b}}$ An increase in revenue per contact sensitivity $\left(\frac{\partial A(\tau)}{\partial \tau}\right)$ leads to an increase in $\tau^{*}$. An intercept-shift in revenue per contact $(A(\tau))$ leads to an increase in $V_{c e}$. It also leads to a decrease in $\tau^{*}$ when $\tau^{*}$ is small to moderate, and an increase in $\tau^{*}$ when it is large. A lower-bound to the cut-off level is: $\tau^{*} \eta_{\bar{S}}=\tau^{2}+1$.

\section{Proof See Appendix B.3.}

The impact of $\mathrm{A}(\tau)$ on $V_{c e}$ follows from the envelope theorem. In terms of the impact of $\mathrm{A}(\tau)$ and $\frac{\partial A(\tau)}{\partial \tau}$ on $\tau^{*}$, the proof in Appendix B.3 is longer than for the previous proposition because of the effects of $A$ on $P$. The essence of the proposition is, however, straightforward. When the sensitivity of revenue to longer $\tau$ increases, there is a pressure towards longer $\tau^{*}$. In case of a positive interceptshift, there is a tendency for the firm to take advantage of the shift by harvesting the database.

\subsection{Change in acquisition rate $(\mathrm{g})$}

Proposition $\mathbf{3}_{\mathbf{c}}$ An increase in acquisition rate leads to a decrease in $\tau^{*}$ and an increase in $V_{c e}$.

Proof The proof is similar to the proof of Proposition 3 a. Since $\bar{S}$ is a linear function of $g$, the elasticity $\eta_{\bar{S}}$ does not depend on $g$. The only term in the $F O C$ that depends on the acquisition rate is the gross margin (through $\bar{S}$ ). Thus all we are interested in is the sign of:

$$
\frac{\partial G M}{\partial g}=\frac{\partial}{\partial g} \frac{R(\tau) \cdot \bar{S}(\tau)-F C}{R(\tau) \cdot \bar{S}(\tau)}=\frac{F C}{R(\tau) \cdot \bar{S}(\tau)^{2}} \frac{\partial \bar{S}(\tau)}{\partial g}
$$

Hence, $\frac{\partial G M}{\partial g}>0$ since $\frac{\partial \bar{S}(\tau)}{\partial g}=\frac{\tau}{1-P(\tau)}>0$. Thus, given that $G M$ acts as a multiplier to $\eta_{D}$, which is negative, an increase in acquisition rate - through increased acquisition spending or increased acquisition effectiveness - will lead to a decrease in $\tau^{*}$ and conversely for a decrease in acquisition rate. Finally, the increase in $V_{c e}$ follows from the envelope theorem and the fact that $\frac{\partial V_{C E}}{\partial g}$ is positive.

This relationship between $g$ and $\tau^{*}$ is a direct result of treating customers as a resource. When the resource is plentiful ( $g$ is high) the firm can harvest it; setting a low $\tau^{*}$. When the resource is scarce ( $g$ is low), the firm must conserve it; setting a high $\tau^{*}$. 


\subsection{Change in discount rate $(\mathrm{r})$}

Proposition $\mathbf{3}_{\mathbf{d}}$ An increase in discount rate leads to an increase in $\tau^{*}$ and a decrease in $V_{c e}$.

Proof Although counterintuitive with regards to $\tau^{*}$, Proposition $3_{\mathrm{d}}$ is straightforward to prove. To show that $\tau^{*}$ increases in $r$, we first note that $\eta_{R}, \eta_{\bar{S}}$, and $G M$ are independent from $r$. Hence, a change in discount rate will only affect $\eta_{D}$. This change is:

$$
\frac{\partial \eta_{D}}{\partial r}=\frac{\partial}{\partial r} \frac{-r \tau}{e^{r \tau}-1}=\frac{\tau}{e^{r \tau}-1}\left(\frac{r \tau e^{r \tau}}{e^{r \tau}-1}-1\right)>0 \forall r>0, \tau>0 .
$$

The derivative of $\eta_{D}$ with respect to $r$ is positive for all positive $r$ and $\tau$. ${ }^{2}$ This implies that the optimal reaction for a firm faced with an increase in discount rate is to increase its inter-communication interval. This may seem counter-intuitive at first, as one might believe that increasing $r$ makes future revenues less attractive and, thus, one might want to decrease $\tau$ so as to realize more profits in the short-term.

What actually happens is that an increase in $r$ leads to a decrease in the discount multiplier $(D)$. This decrease means that, holding everything else constant, the value of each name decreases, and so does the value of the database. This decrease must be offset by either an increase in database size $(\bar{S})$ or an increase in expected profit per name per communication $(R)$. Both are accomplished by increasing $\tau$.

It is straightforward to show that as $r$ increases, the value of the database decreases. Applying the envelope theorem one more time we have:

$$
\frac{\partial V_{c e}}{\partial r}=(R \bar{S}-F C) \frac{\partial}{\partial r} \frac{e^{r \tau}}{e^{r \tau}-1}=(R \bar{S}-F C) \frac{-\tau e^{r \tau}}{\left(e^{r \tau}-1\right)^{2}}<0 .
$$

Hence, an increase in $r$ leads to both an increase in optimal inter-communication time $\left(\tau^{*}\right)$ and a decrease in database value $\left(V_{c e}\right)$. The impact of $r$ on $\tau^{*}$ could be used to study how a firm should vary its communication time in response to interest rate variations. However, a more interesting study is how $\tau^{*}$ changes as a firm grows from a start-up to a large legitimate company. Indeed, a start-up is a risky venture and its implicit discount rate will be high. As the firm grows, its future becomes less uncertain and its discount rate diminishes. Proposition $3_{\mathrm{d}}$ states that in such case, the firm will decrease its inter-communication time as it grows. This decrease in $\tau$ effectively shifts the firm from a "database growth" regime to a "profit generation" regime as it now tries to extract more revenues from its database and leaves itself less time between communication to replace the names lost.

\subsection{Changes in costs}

The firm's reaction to any increase in cost, whether fixed or variable, is to increase optimal inter-communication interval. The intuition for this is straightforward. An

\footnotetext{
$\overline{2 \text { To see this, note that at }} \tau=0, r \tau e^{r \tau}=e^{r \tau}-1=0$, and for all $\tau>0, \tau>0, \frac{\partial}{\partial \tau} r \tau e^{r \tau}=r e^{r \tau}$ $+r^{2} \tau e^{r \tau}>\frac{\partial}{\partial \tau} e^{r \tau}-1=r e^{r \tau}$.
} 
increase in cost reduces per-campaign profits. The firm must then increase $\tau^{*}$ to try to boost its profits per campaign - reducing the number of communications sent raises expected revenue per campaign.

Proposition $\mathbf{3}_{\mathbf{e}}$ An increase in fixed or variable costs leads to a decrease in $V_{c e}$ and an increase in $\tau^{*}$.

Proof Comes from $\frac{\partial V_{c e}}{\partial F C}<0, \frac{\partial V_{c e}}{\partial V C}<0, \frac{\partial \eta_{R}}{\partial V C}>0$, and $\frac{\partial \mathrm{GM}}{\partial}<0$.

\subsection{Take away}

The study of the levers of customer value reveals some consistent patterns. The firm has two conflicting objectives that it is trying to balance: database growth and database harvesting. Database growth involves a long-term approach, so the marketer needs to adjust marketing actions so as to increase the database size, $(\bar{S})$. In the $C E$ framework this can be achieved by reducing the communication frequency (i.e., increasing $\tau$ ). On the other hand, when the objective is to harvest the database, marketing actions need to be adjusted so as to increase short-term revenues. This can be achieved by reducing the inter-communication interval (i.e., decreasing $\tau$ except in some occasions when $\tau$ is very small, in which case both objectives are achieved by increasing $\tau$ ).

Whenever there is a change in the firm's performance or in the environment that is beneficial to the firm (e.g., higher return from acquisition, better retention rate, lower interest rate), the firm can adjust its actions in a way that favors harvesting at the expense of growth (i.e., decrease $\tau$ ). Conversely, if the change is detrimental (e.g., lower return per campaign, higher costs), the firm must counteract this change by leaning more toward growth rather than harvesting (i.e., increase $\tau$ ).

\section{Acquisition policy}

Now that we have discussed the key levers of the database value and can maximize the marketing actions $\left(\tau^{*}\right)$ for any given acquisition policy, we can turn to the problem of optimizing acquisition. Indeed, we have thus far assumed that acquisition expenditures were fixed. We were able to do this since Lemma 2 shows that the actual spending on name acquisition is separable from the optimization of $\tau$, and therefore only the acquisition rate matters for the derivation of the optimal intercommunication interval. The implication of this result is that the marketer first needs to derive the optimal $\tau$ for a given acquisition rate (Section 3.1), and then optimize acquisition spending given the solution for $\tau^{*}$. Section 4 ignored acquisition except for to show that an increase in $g$ leads to a decrease in $\tau^{*}$ (Proposition $3_{\mathrm{c}}$ ).

\subsection{Optimal acquisition policy}

Our goal for this section is to derive the optimal acquisition policy $\left(g^{*}\right)$ as a function of the optimal marketing policy $\tau^{*}$. We found in Section 4.3 that a higher customer 
acquisition rate leads to greater database size, and larger revenues, but these benefits must be offset against the cost of acquiring these customers. It is critical, for this analysis, to understand that the acquisition costs correspond to a stream of payment over time, rather than a one-time transaction.

Formally, since, by Proposition 2, we can separate the optimization of the optimal inter-communication interval $(\tau)$ from the optimal acquisition rate, then $\tau^{*}$ can be expressed as a function of acquisition spending. Thus we can find the optimal expenditure on acquisition by taking the derivative of $C E$ with respect to $g$. The optimum is found when marginal revenues are equal to marginal costs. We formalize this in the following proposition:

Proposition 4 If the periodicity at which the acquisition expenditures are recognized is equal to the periodicity of the communications, then the optimal acquisition rate occurs when the marginal acquisition cost per name is equal to the CLV of the name.

\section{Proof See Appendix C.}

The idea behind the proof is straightforward. Let us assume that the firm currently acquires $g$ customers per period for a cost of $A Q(g)$ and is considering acquiring one more. This will cost of $\triangle A Q=A Q(g+1)-A Q(g)$. This is worth doing if the net present value $(N V P)$ of the additional expenses is lower than the $N P V$ of the additional revenues. In each period, the cost of acquiring the extra customer is $\triangle A Q$ and the $N P V$ of the future revenues derived from the acquired customer is its $C L V$. Thus, one will acquire customers as long as their acquisition costs are lower than their CLV.

It may seem odd that our analysis shows on the one hand that maximizing $C L V$ is sub-optimal for the firm, and on the other hand that $C L V$ is the appropriate to set customer acquisition policies. There seems to be a disconnect here as if we set $\tau^{*}$ according to our Customer Equity framework, the $C L V$ of each of the customers in our database is by definition smaller than if we set $\tau^{*}$ so as to maximize the CLV. Thus, since we have just shown that acquisition is set based on the $C L V$ of the people we acquire, we could spend more on acquisition, and thus acquire more customers, if we set $\tau^{*}$ according to the $C L V$ rather than the $C E$. It stands to reason that if maximizing $C L V$ leads to customers that are worth more than maximizing $C E$, and that maximizing $C L V$ allows the firm to acquire more such customers, than maximizing should be more profitable than maximizing $C E$. Why is it not the case? To understand why, let us analyze a synthetic case where we set communication and acquisition policies according to both $C E$ and $C L V$ and see how the firm's decision and its profits change depending on whether it maximizes $C L V$ or $C E$.

To specify the various parameters of our synthetic case, let us assume that there are no fixed costs of communications $(F C=0)$. This insures that any differences we find are due to the revenue and acquisition stream and not due to an allocation of fixed costs over a smaller or larger pool of customers. Further, let us use quadratic acquisition costs $\left(A Q(g)=g^{2}\right)$, revenues per communication that follow an exponential recovery process $\left(R(\tau)=r_{0}\left(1-e^{-r i \tau}\right)\right.$, where $\mathrm{r}_{0}=5$ and $\left.\mathrm{r}_{1}=10\right)$, and a retention rate that follows a $\log$-logistic function $\left(P(\tau)=a \lambda(\lambda \tau)^{a-1} /\left(1+(\lambda \tau)^{a}\right)^{2}\right.$, where $\alpha=2$ and $\lambda=3 / 4)$. These functional forms are arbitrary, they are chosen as simple widely used expressions that conform to the assumptions made in Section 2. 
Finally, let us assume that the firm is a start-up and thus use a relatively high discount rate of $20 \%$ per annum (Haenlein et al. 2006).

Using these equations and the solver function in excel, it is straightforward to find the $\tau$ that maximizes the $C E\left(\tau_{C E}^{*}\right)$ and compare it to the $\tau$ that maximizes the $C L V$ $\left(\tau_{C L V}^{*}\right)$. We can then compute the $C E$ that would be generated if one used $\tau_{C L V}^{*}$ rather than $\tau_{C E}^{*}$. This static comparison will help us better understand the ramification of maximizing $C L V$ rather than $C E$.

The results of the maximization with regards to $C E$ and $C L V$ are shown in Table 1 . As one can see, maximizing $C E$ leads to an inter-communication interval that is $13 \%$ larger than if one were to maximize the $C L V$. This leads to a slightly larger retention rate and larger revenue per communication per customer, but as expected a lower $C L V$ (1\% smaller). And thus smaller acquisition budgets $(-2 \%)$ and fewer new customers acquired every year $(-1 \%)$. However, because the retention rate is larger and fewer communications are sent with the larger $\tau$ when maximizing $C E$, this approach results in a larger pool of customers at any point in time $(+13 \%)$. This leads to a higher value of the firm $(+2 \%)$ despite the fewer opportunities to generate revenues $(-12 \%$ contacts per year).

This shows the problem with maximizing $C L V$ as a business practice. $C L V$ favors generating profits now at the expense of future profits. This is rational as future profits are worth less than current ones. However, this comes at the expense of lower retention rates. This is factored in the $C L V$ framework on a cohort by cohort basis. However, what the $C L V$ framework does not take into account is that lower retention rates don't just mean that the firm will extract less revenue from newly acquired customers in the future, they also mean that the firm currently has fewer of its past customers to extract revenue from in the present.

To better understand how $C L V$ the impact of past cohorts on present revenues, consider Fig 1a, b. Figure 1a represents the evolution of one cohort. Figure 1b represents the make up of the database as new cohorts are acquired and old ones shrink. Focusing on the tenth year (Y10), one can see that at any point in time, the database contains the names acquired as part of the latest cohort, plus the names

Table 1 Static comparison of CE and CLV maximizing strategies

\begin{tabular}{lrrrr}
\hline & CE & CLV & Diff & \% Diff \\
\hline Optimal communication interval $\left(\tau^{*}\right)$ & 0.802 & 0.708 & 0.094 & $13.33 \%$ \\
Retention rate $(\mathrm{P})$ & 0.487 & 0.485 & 0.002 & $0.39 \%$ \\
Revenue per communication per customer & 4.998 & 4.996 & 0.003 & $0.05 \%$ \\
Non-discounted lifetime revenues & 9.734 & 9.694 & 0.041 & $0.42 \%$ \\
Customer lifetime value (CLV) & 8.623 & 8.703 & -0.080 & $-0.92 \%$ \\
Number of customers acquired per year $(\mathrm{g})$ & 4.311 & 4.351 & -0.040 & $-0.92 \%$ \\
Yearly acquisition spending $(\mathrm{AQ})$ & 18.589 & 18.935 & -0.347 & $-1.83 \%$ \\
Steady state customer base $(\bar{S})$ & 6.734 & 5.975 & 0.759 & $12.70 \%$ \\
Profit per communication & 33.657 & 29.848 & 3.808 & $12.76 \%$ \\
\# of communications per year & 1.247 & 1.413 & -0.166 & $-11.76 \%$ \\
Value of the firm $(\mathrm{CE})$ & 135.898 & 132.989 & 2.909 & $2.19 \%$ \\
\hline
\end{tabular}


from the previous cohort that survived one period, plus the names of the cohort before that survived two periods, and so on. Hence, by collating at any one time the individuals retain across cohorts we can recreate the profile of any single cohort across time. Thus, the revenues generated by the database in each period are equal to the undiscounted revenues generated by one cohort over its entire lifetime. To maximize profits for a given acquisition policy, the firm should maximize the undiscounted cash flow generated from an individual rather than the discount cash flow (i.e., the $C L V$ ). Referring back to Table 1 , we can see that although the $\tau_{C L V}^{*}$ produces a higher CLV than $\tau_{C E}^{*}$, it does produce a lower non-discounted lifetime revenues (by $0.42 \%$ ) which, coupled with a smaller steady state customer base, yields a lower value for the firm.

A side benefit of the fact that at any point in time the profile of the customer base is equivalent to the profile of a cohort over its lifetime is that it allows us to relax the assumptions of constant retention rate and revenues. Indeed, looking at Fig. 2a we see a cohort profile where retention is not constant over the life of its members. Retention is low in the first year, high for the next 4 years, then steady for the remaining years. This uneven retention violates our assumption of constant retention rate which is necessary to simplify the infinite sum of future revenues into the simple Equation that is (9). However, as is demonstrated by Figure 2b, the database size in this case is still equal to the sum of the cohort size over its life, and the revenues generated by the database in one period are still equal to the revenues generated by a cohort over its life. Thus, Lemma 1 will still hold in that there is a fixed long-term size to the database (the actual size will not be $\frac{\tau g}{1-P(\tau)}$ as $P$ is not constant over time, but it can still be calculated, we show an example of this in Section 6.1). Further, one can still use the first order condition developed in (12) to maximize the database value. Finally, proposition 4 still applies, and we can compute the maximum acquisition spending by computing the $C L V$ of each cohort.

\subsection{The path to steady state}

Skeptics might argue that our Customer Equity framework might work well for an established company that already has a customer base and does indeed derive revenues from both its new and old customers. But, what of a start-up company? It does not have 'old' customers. Wouldn't a $C L V$ maximization approach allow it to spend more on customer acquisition and thus grow faster?

To show this is not the case and that the $C E$ maximization approach primes over a $C L V$ approach even away from steady state, we use the synthetic example developed in the preceding section and plot the growth in database size and revenues over time for our hypothetical firm assuming it starts with no customer at time 0. As one can see in Fig. 3, the $C E$ approach grows the customer base faster and to a higher level than the $C L V$ approach. Revenues follow a similar pattern.

As a bonus, the start-up firm will need to invest less in customer acquisition to reach the faster growth. Thus, it will need to raise less capital and provide a larger $R O I$ than if it were to follow a $C L V$ maximization strategy. These two benefits should make raising capital easier. 

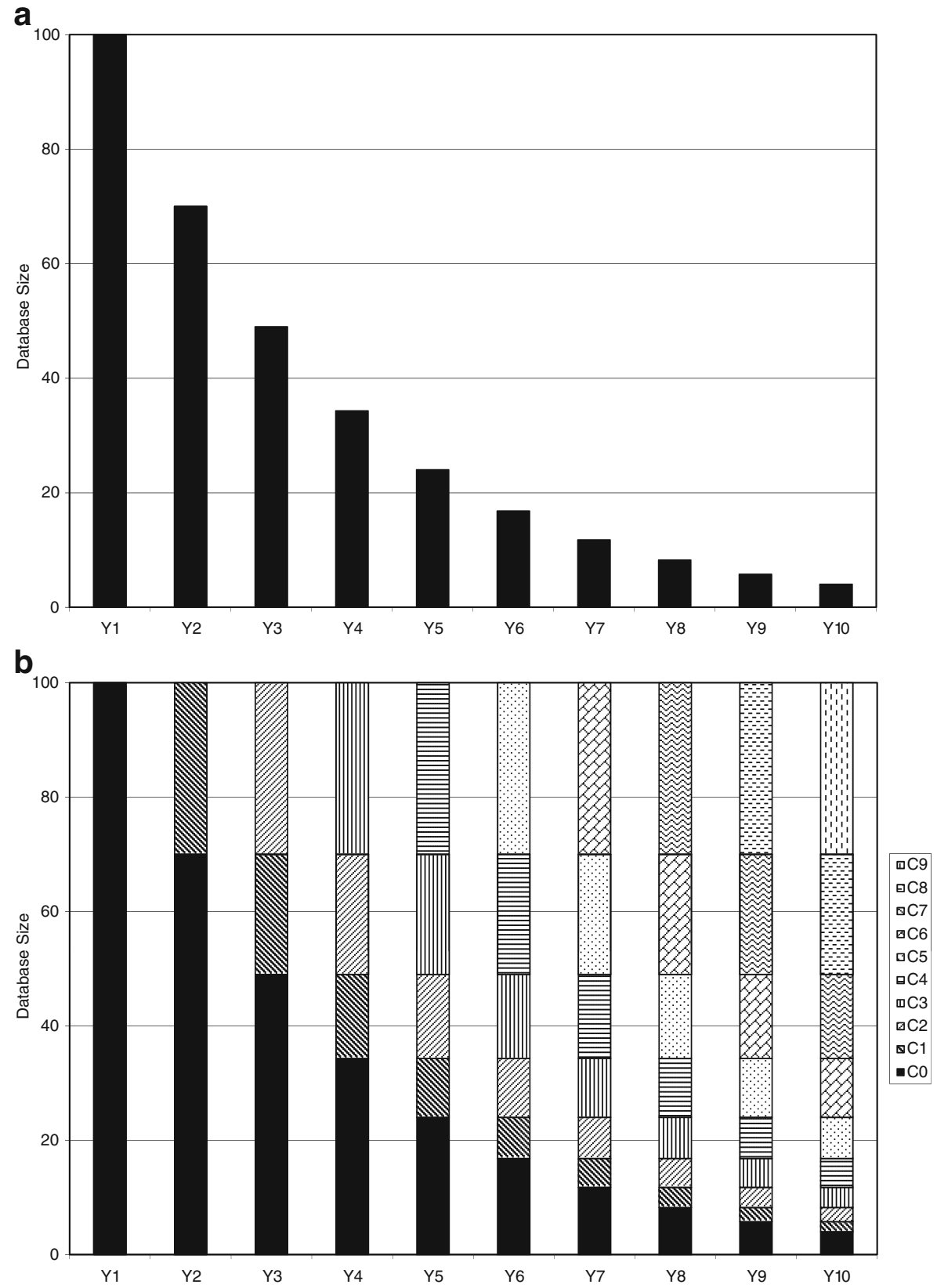

Fig. 1 a Cohort Size Over Time b Database Size Over Time (by cohorts) 


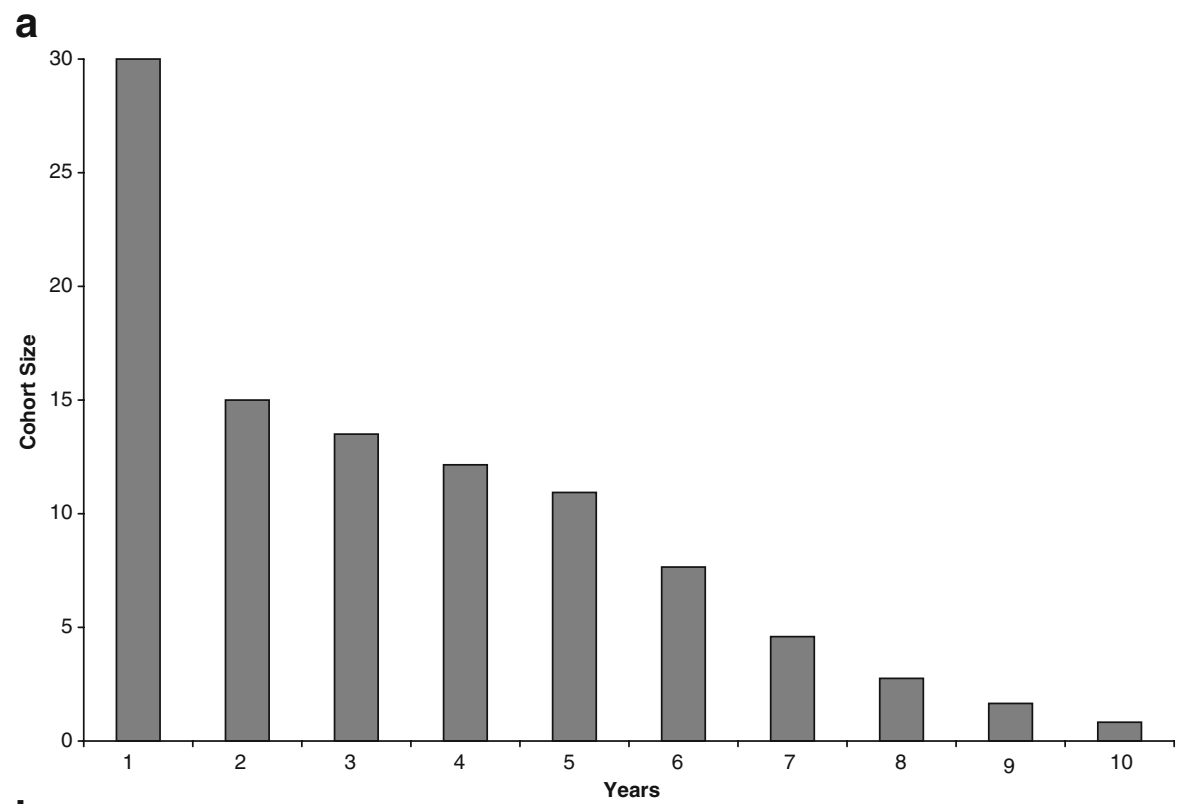

b

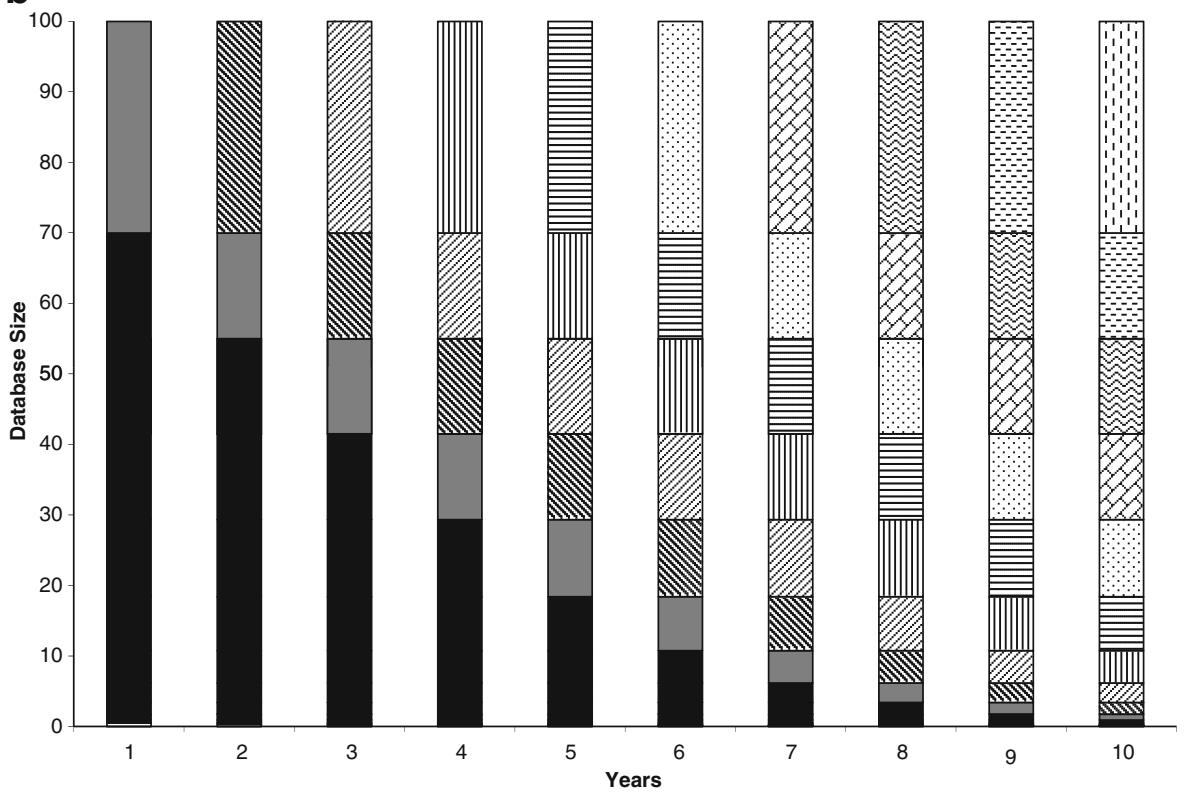

Fig. 2 a Cohort Size- Uneven Attrition b Database Size- Uneven Attrition

\section{Heterogeneity}

An important assumption was made about customers being identical in terms of key constituents of the $C E$ : the revenue function, the retention function, and acquisition policy. We assumed that the database value could be expressed as a function of an 


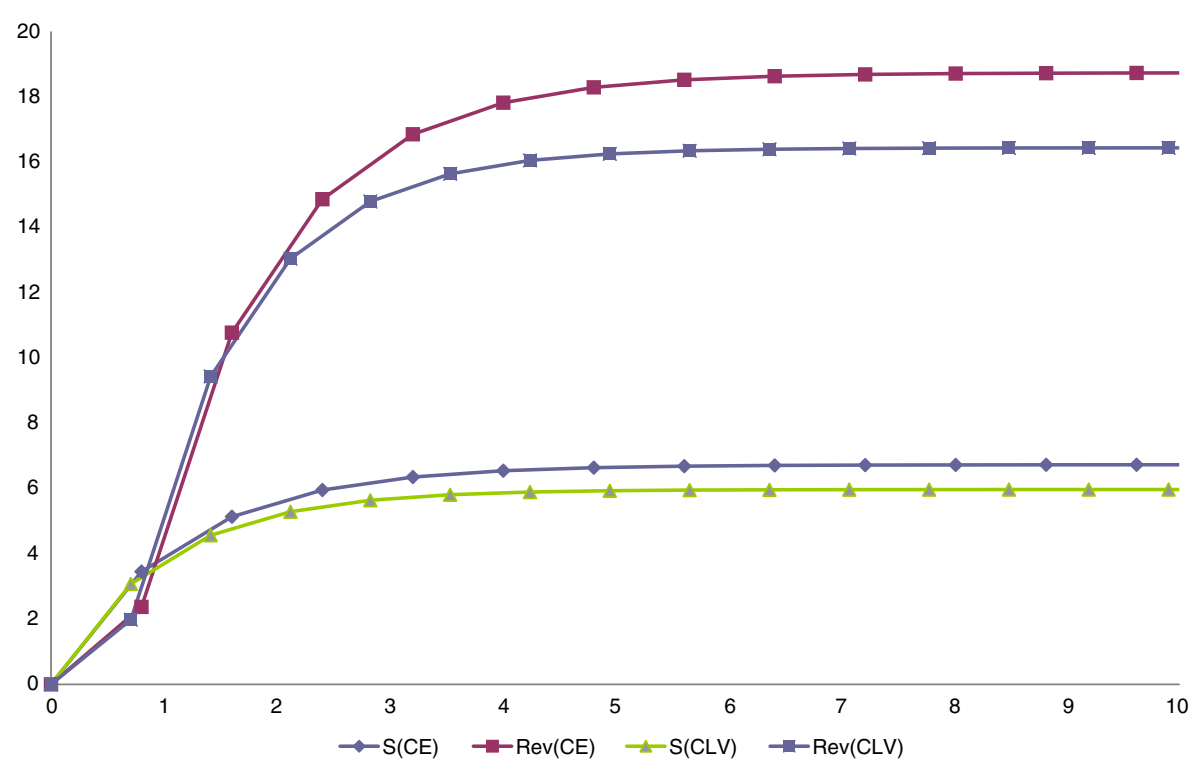

Fig. 3 Customer base size and revenues

"average" consumer. This simplified view the derivation of many of the results, but we need to understand how robust the results are if we relax this assumption.

First note that marketers deal with two types of heterogeneity: observed and unobserved. This leads to two questions. First, what happens when customers are treated as identical when they are not (unobserved heterogeneity)? Second, how does our framework change, and is it still applicable, when heterogeneity is observed and the marketer treats different customers differently by sending different communications to different groups of customers, or send communications at different rates to different customers?

\subsection{Unobserved heterogeneity}

Customers can be heterogeneous in their retention probability $(P)$ and/or their expected return from each communication $(R)$. If consumers have identical retention probabilities, but are heterogeneous in their expected return, then, if all customers are treated as being identical, the average expected return $(\bar{R})$ can be used to maximize profits. Indeed, let $f(R)$ be the probability density function of $R$. The expected database value is obtained by integrating out customer equity across customers:

$$
E\left[V_{c e}\right]=\int(R \bar{S}-F C) \frac{e^{r \tau}}{e^{r \tau}-1} f(R) d R=(\overline{R S}-F C) \frac{e^{r \tau}}{e^{r \tau}-1} .
$$

Taking the same approach is a little more complicated for heterogeneity in the retention rate. Indeed, $P$ appears on the denominator of $C E$ through $\bar{S}$. Thus, going back to Little's Law, we need to compute:

$$
\bar{S}=\tau \cdot g \cdot E\left[\frac{1}{1-P}\right] \neq \frac{\tau \cdot g}{1-E[P]}
$$


The difference stems from the fact that customers with higher retention rates stay in the database longer than customers with lower retention rates. For instance, if we assume that the acquisition efforts yield a stream of customers whose retention rate has a Beta distribution, $\mathrm{B}(a, \beta)$ with $\beta>1,{ }^{3}$ then we have:

$$
\bar{S}=\tau g \int_{0}^{1} \frac{f(P)}{1-P} d P=\tau g \int_{0}^{1} \frac{\Gamma(a+\beta)}{\Gamma(a) \Gamma(\beta)} p^{a-1}(1-P)^{\beta-2} d P .
$$

Since $\Gamma(n+1)=n \Gamma(n)$, we have:

$$
\bar{S}=\tau g \frac{(a+\beta-1)}{(\beta-1)} \int_{0}^{1} \underbrace{\frac{\Gamma(a+\beta-1)}{\Gamma(a) \Gamma(\beta-1)} p^{a-1}(1-P)^{\beta-2} d P=\frac{\tau . g}{1-\frac{a}{(a+\beta-1)}}}_{=1}
$$

where we recognize the term $\frac{a}{a+\beta-1}$ as the expected value of a $B(a, \beta-1)$. Hence, if the heterogeneity in retention rate in the acquisition stream is characterized by a $\mathrm{B}(\mathrm{a}$, $\beta)$, then the marketer should optimize its database using the expected value of a $B$ $(a, \beta-1)$ as its average $P$.

\subsection{Observed heterogeneity}

A firm faced with observed heterogeneity can adapt its marketing actions in two ways. The traditional method used by marketers is to segment customers into mutually exclusive segments based on their purchase behavior and then treat each customer segment as homogenous. Airlines, with their tiered frequent flyer programs, are classic examples of this approach. Proponents of Customer Relationship Management (CRM) propose a more radical approach where each customer is able to receive an individually tailored marketing communication.

It is easy to see that the $C E$ framework is appropriate for the traditional customer segmentation approach. One can optimize each segment's marketing communications and overall value separately, as a function of each segment's acquisition, margins, and retention rates. One can also easily compute the benefits of having more or less segments by comparing the segment values in an $N$ segment world to those in an $N+1$ segment world. The trade-off one makes when increasing the number of segments is that revenues should increase with the number of segments as one can better customize the marketing communications when the segments become more homogenous; but, communication costs will also increase as different communication must be created for different segments. This naturally leads to an optimal number of segments.

The promises of $C R M$ are to make the customization costs so low that it now becomes feasible to have segments of size one. If this promise were realized, one could argue that a $C L V$ model is more appropriate than a $C E$ model. Indeed, if segments are of size one then they truly are depletable. When the segment member defects; the segment is depleted. Newly acquired customers constitute

\footnotetext{
$\overline{{ }^{3} \beta>1 \text { is needed to ensure that }} f(1)=0$. If $f(1)>0$ then some people will stay in the system forever, regardless of the firm's actions. This would lead to degenerate solutions.
} 
their own segments rather than replenishing pre-existing segments. If all decision variables (acquisition spending, communication frequency, communication content, etc.) are optimized at the individual level independent of other individuals, then the $C L V$ approach might be the correct one. However, that is not what $C R M$ actually entails.

What $C R M$ proposes to do in practice is to develop a set of rules or models that, when applied to the information known about the firm's customer, yield a customized marketing message (Winer 2001). The nature and the amount of customization varies across applications. For instance, American Airlines uses CRM to select which WebFares to advertise to each of its frequent flyers in its weekly emails. Citibank uses $C R M$ to direct callers to its toll free lines to the sales person that is most adept at selling the type of products that their predictive model selected as a likely candidate for cross-selling. Amazon.com uses $C R M$ to try to up-sell its customers by offering them bundles of books they might be interested in. In each of these examples, different customers will experience different offerings. Different frequent flyers will be alerted of different promotional fares depending on the cities they fly most; different readers will be offered different book bundles depending on the books they are searching for. Nevertheless, the set of rules or models that are used to generate these offers will be identical for all frequent flyers, or all Amazon customers. The output will be different, but the process will be identical. Further, the costs of creating the rules and communication templates will be shared across all individuals rather than borne individually. As such, the $C E$ framework developed here still applies. The $C R M$ objectives will be to develop the set of rules that maximize overall $C E$ rather than any specific individual's $C L V$. When doing so, it is critical to use the appropriate objective function. This will be achieved by considering the impact of the $C R M$ efforts on retention and amounts spent and maximize the long term database value just as has been done in this paper with the inter-communication interval $(\tau)$.

\section{Managerial implications and conclusion}

The lifetime value of a firm's customer base depends both on the acquisition process used by the firm and the marketing actions targeted at these customers. To take both aspects into account, we draw on the theory of optimal resource management to revisit the concept of customer lifetime value. Our contention is that customers are a renewable resource and hence marketing actions directed at acquiring and extracting revenues from customers should take a long-term value-maximization approach. Thus we study the implications of moving from Customer Lifetime Value maximization to Customer Equity maximization. In answer to the first questions raised in the introduction (Does $C L V$ maximization equate to $C E$ maximization?), our findings indicate that disregarding future acquisition leads to sub-optimal revenue extraction strategies (Proposition 1). This will then lead to sub-optimal acquisition strategies as acquisition spending are set according to revenues (Proposition 4).

Following on from this, our second research question addresses what is the proper benchmark to use to guide customer acquisition policy. We find that the firm is able to generate more profits by spending less on acquiring a new customer if it were to use the $C E$ approach, than if it were to use a $C L V$ approach. 
To answer the final research questions raised (What is the appropriate metric, how is it computed and maximized?), we first need to consider how the database value is impacted by actions of the marketer. As we summarized in Proposition 2, our model of customer equity directly accounts for the impact of marketing actions on the value of a firm's customer assets. This allows us to derive the optimal actions for the firm, and we derive the long-term steady-state size of a firm's customer base (Lemma 1). Our first-order condition for the maximization of customer equity (Proposition 2) shows that a firm should adapt its marketing actions to changes in the critical factors that impact database value. The strength of the first-order condition derived in Proposition 2 is that it is easy to optimize empirically. The firm can run a series of tests to estimate the various elasticities and infer from them if it should increase or decrease its communication periodicity. This is simplified by the fact that customer equity has a unique maximum. The first-order condition also lends itself well to static comparisons (Propositions $3_{\mathrm{a}}$ to $3_{\mathrm{d}}$ ). This exercise shows how firms are attempting to balance two conflicting objectives: database growth and database harvesting. Whenever there is a change in the firm's performance or in the environment that is beneficial to the firm (e.g., higher return from acquisition, better retention rate, lower interest rate), the firm should adjust its actions in a way that favors harvesting at the expense of growth. Conversely, if the change is detrimental (e.g., lower return per campaign, higher costs), the firm must counteract this change by leaning more toward growth rather than harvesting.

We finish the paper with a discussion of customer heterogeneity. We show that our model is robust to unobserved heterogeneity. The learning point there is that ignoring heterogeneity in customers' valuation of the communication is less of an issue than ignoring customer heterogeneity in retention rate.

We recognize several limitations inherent in this study. We made specific assumptions regarding the relationship between customer retention and the firm's communication strategy, both in terms of timing (through $\tau$ ) and in content (through the relationship between $A$ and $P$ ) that could be relaxed in future work. Further work also needs to be done to incorporate the impact of competitive actions and reactions on the measurement of customer equity. This might potentially be done by folding our approach with a theoretical model such as the one developed by Fruchter and Zhang (2004). We believe, however, that the framework presented in this study is a useful starting point for such a competitive analysis.

Open Access This article is distributed under the terms of the Creative Commons Attribution Noncommercial License which permits any noncommercial use, distribution, and reproduction in any medium, provided the original author(s) and source are credited.

\section{Appendix A: proof of lemma 1 and proposition 1}

\section{A.1 Proof of Lemma 1}

Let $P(\tau)$ be the proportion of the database that is retained from one campaign to the next, given that $\tau$ is the fixed inter-campaign time interval. Let the acquisition stream be $g(\tau)=\tau$. $g$. If the firm begins with a database size at $S_{0} \neq \bar{S}$, we show that the long- 
term stationary value of the database is still $\bar{S}$. To find this value, solve the Law of Motion for the size of the database as:

$$
\bar{S}=\bar{S} \cdot P(\tau)+\tau \cdot g=\frac{\tau \cdot g}{1-P(\tau)} .
$$

What if the database size is not at the stationary value? Then, if $\tau$ is constant, the state variable converges to the stationary value. To see this, pick any arbitrary value for the starting size of the database e.g. pick any $\varepsilon \neq 0$ such that $S_{i}<\bar{S}$ or $S_{i}>\bar{S}$ e.g.:

$$
S_{i}=\frac{\tau \cdot g}{1-P(\tau)}+\varepsilon
$$

so that for the next period:

$$
S_{i+1}=S_{i} P(\tau)+\tau . g=\left(\frac{\tau . g}{1-P(\tau)}+\varepsilon\right) P(\tau)+\tau . g=\frac{\tau \cdot g}{1-P(\tau)}+\varepsilon \cdot P(\tau)
$$

and for any $\mathrm{n}>0$,

$$
S_{i+n}=\frac{\tau \cdot g}{1-P(\tau, k)}+\varepsilon P(\tau)^{n}
$$

and $\lim _{n \rightarrow \infty} S_{i+n}=\bar{S}$, since $P(\tau) \in(0,1)$ and therefore $\varepsilon P(\tau)^{n} \rightarrow 0$ as $n \rightarrow \infty$.

A.2 maximization of customer value versus customer equity

Assume that we look at the equilibrium conditions so that $S_{i}=\bar{S}$. The value of an individual customer name is defined as:

$$
V_{c l v}=\sum_{i=0}^{\infty} e^{-i r \tau} P(\tau)^{i}\left(R(\tau)-\frac{F C}{\bar{S}}\right)=\left(R(\tau)-\frac{F C}{\bar{S}}\right) \frac{e^{r \tau}}{e^{r \tau}-P(\tau)}
$$

The database value, is defined as:

$$
V_{c e}=\sum_{i=0}^{\infty} e^{-i r \tau}(P(\tau) \bar{S}-F C)=(R(\tau) \bar{S}-F C) \frac{e^{r \tau}}{e^{r \tau}-1} .
$$

Hence, we have the database value as a function of the customer value:

$$
V_{c e}=V_{c l v} \bar{S} \frac{e^{r \tau}-P(\tau)}{e^{r \tau}-1} .
$$

To maximize, we differentiate with respect to $\tau$ :

$$
\frac{\partial V_{c e}}{\partial \tau}=\bar{S} \frac{e^{r \tau}-P(\tau) \partial V_{c l v}}{e^{r \tau}-1}+V_{c l v} \frac{\partial}{\partial \tau} \bar{S} \frac{e^{r \tau}-P(\tau)}{e^{r \tau}-1} .
$$

Hence, maximizing the $C L V$ and the $C E$ will be identical iff:

$$
\underbrace{\frac{\partial V_{c e}}{\partial \tau}}_{0 ?}=\bar{S} \underbrace{\frac{e^{r \tau}-P(\tau)}{e^{r \tau}-1}}_{\neq 0} \underbrace{\frac{\partial V_{c l v}}{\partial \tau}}_{=0}+\underbrace{V_{c l v}}_{\neq 0} \underbrace{\frac{\partial}{\partial \tau} \bar{S} \frac{e^{r \tau}-P(\tau)}{e^{r \tau}-1}}_{=0 ?}=0 .
$$

The fourth term $\left(\frac{\partial}{\partial \tau} \bar{S} \frac{e^{r \tau}-P(\tau)}{e^{r \tau}-1}\right)$ will be equal to 0 iff: $\eta_{\bar{S}}+\eta_{\frac{e^{r \tau}-P(\tau)}{e^{r \tau}-1}}$, or $\eta_{\bar{S}}=-\eta_{\frac{e^{r \tau}-P(\tau)}{e^{r \tau}-1}}$. 
We know that $1+\frac{\partial P(\tau)}{\partial \tau} \frac{\tau}{1-P(\tau)}$, we now need to compute $\frac{\eta_{e^{r \tau}-P(\tau)}}{e^{r \tau}-1}$ :

$$
\begin{aligned}
\frac{\partial}{\partial \tau} \frac{e^{r \tau}-P(\tau)}{e^{r \tau}-1} & =\frac{r e^{r \tau}-\frac{\partial P(\tau)}{\partial \tau}}{e^{r \tau}-1}-\frac{\left(e^{r \tau}-P(\tau)\right) r e^{r \tau}}{\left(e^{r \tau}-1\right)^{2}} \\
& =\frac{1}{e^{r \tau}-1}\left(r e^{r \tau}-\frac{\partial P(\tau)}{\partial \tau}-\frac{\left(e^{r \tau}-P(\tau)\right) r e^{r \tau}}{e^{r \tau}-1}\right)
\end{aligned}
$$

Thus:

$$
\begin{aligned}
\eta_{\frac{e r \tau-P(\tau)}{e^{r \tau}-1}} & =\frac{1}{e^{r \tau}-1}\left(r e^{r \tau}-\frac{-\partial P(\tau)}{\partial \tau}-\frac{\left(e^{r \tau}-P(\tau)\right) r e^{r \tau}}{e^{r \tau}-1}\right) \frac{\tau}{\frac{e^{r t}-P(\tau)}{e^{r t}-1}} \\
& =-\left[\tau r e^{r \tau}\left(\frac{1-P(\tau)}{\left(e^{r \tau}-1\right)\left(e^{r \tau}-P(\tau)\right)}\right)+\frac{\partial P(\tau)}{\partial \tau} \frac{\tau}{e^{r \tau}-P(\tau)}\right] .
\end{aligned}
$$

This means that $\eta_{S}$ and $-\eta_{\frac{e^{r \tau}-P(\tau)}{e^{r \tau}-1}}$ are both affine transformations of $\frac{\partial P(\tau)}{\partial \tau}$, and thus will be equal for all $\frac{\partial P(\tau)}{\partial \tau}$ iff both their intercepts and their slopes are equal, or:

$$
1=\tau r e^{r \tau}\left(\frac{1-P(\tau)}{\left(e^{r \tau}-1\right)\left(e^{r \tau}-P(\tau)\right)}\right) \quad \text { and } \quad \frac{\tau}{1-P(\tau)}=\frac{\tau}{e^{r \tau}-P(\tau)} .
$$

The second condition gives us that they will be equal only when $\tau=0$. Applying l'Hospital Rule to the first condition we find that, at the limit for $\tau \rightarrow 0$, the first condition is also satisfied. Hence, this shows that it will only be for $\tau=0$ that $\frac{\partial}{\partial \tau} \bar{S} \frac{e^{r \tau}-p(\tau)}{e^{r \tau}-1}$. And thus, maximizing the $\mathrm{V}_{\mathrm{clv}}$ and the $V_{c e}$ lead to the same optimal only when $\tau^{*}=0$, which cannot happen since at $\tau=0$ the database value is negative $\left(\left(V_{c e}(0)=-\infty\right)\right)$. QED

\section{Appendix B: maximum derivation}

We handle the maximization of the database value in three steps. First, we derive the first-order condition that needs to be satisfied for a $\tau$ to be optimal. Second we show that such a $\tau$ exists. And third, we provide conditions under which the maximum is known to be unique.

\section{B.1 First-order condition}

To derive the first-order condition related to the maximization of customer equity with respect to the inter-communication time, we seek the point at which the derivative of (B-1) with respect to $\tau$ is null. We do so in the following steps:

$$
C E(\tau)=(R(\tau) \cdot \bar{S}(\tau)-F C) \frac{e^{r \tau}}{e^{r \tau}-1}-A Q \frac{e^{r}}{e^{r}-1} . \quad(\mathrm{B}-1)
$$


Let $\Omega(\tau)=R(\tau) . S(\tau)$, hence:

$$
\begin{aligned}
\frac{\partial C E(\tau)}{\partial \tau} & =\frac{\partial}{\partial \tau}(\Omega(\tau)-F C) \frac{e^{r \tau}}{e^{r \tau}-1} \\
& =\frac{e^{r \tau}}{e^{r \tau}-1} \frac{\partial \Omega(\tau)}{\partial \tau}+(\Omega(\tau)-F C)\left[\frac{r e^{r \tau}}{e^{r \tau}-1}-\frac{r e^{2 r \tau}}{\left(e^{r \tau}-1\right)^{2}}\right] \\
& =\frac{e^{r \tau}}{e^{r \tau}-1}\left[\frac{\partial \Omega(\tau)}{\partial \tau}+r(\Omega(\tau)-F C)\left(1-\frac{e^{r \tau}}{\left(e^{r \tau}-1\right)}\right)\right] \\
& =\frac{e^{r \tau}}{e^{r \tau}-1} \frac{\Omega(\tau)}{\tau}\left[\eta_{\Omega}+\eta_{D} \frac{\Omega(\tau)-F C}{\Omega(\tau)}\right] .
\end{aligned}
$$

Further, since $\Omega(\tau)=R(\tau) . S(\tau)$, then $\eta_{\Omega}=\eta_{R}+\eta_{\bar{s}}$. Hence:

$$
\frac{\partial C E(\tau)}{\partial \tau}=\frac{e^{r \tau}}{e^{r \tau}-1} \frac{R(\tau) \cdot \bar{S}(\tau)}{\tau}\left[\eta_{R}+\eta_{\bar{S}}+\eta_{D} \frac{R(\tau) \cdot \bar{S}(\tau)-F C}{R(\tau) \cdot \bar{S}(\tau)}\right] .
$$

If we restrict ourselves to cases where the optimal database value is positive (otherwise the firm would not engage in database driven marketing) then we have $\mathrm{R}$ $(\tau)>0$ and $R(\tau) \bar{S}(\tau)-F C>0$ and thus, at the maximum, the following first-order condition needs to be satisfied:

$$
\eta_{R}+\eta_{\bar{S}}+\eta_{D} G M=0
$$

Where $G M=\frac{R(\tau) \cdot \bar{S}(\tau)-F C}{R(\tau) \cdot \bar{S}(\tau)}$ is the gross margin generated by each communication.

The technical appendix contains the proof of the existence and uniqueness of the maximum.

B.2 change in retention probabilities

i. Change in $\partial P(\tau) / \partial \tau$

The retention sensitivity $(\partial P(\tau) / \partial \tau)$ only affects the FOC through $\eta_{\bar{S}}$. An increase in retention sensitivity will lead to an increase in $\eta_{\bar{S}}$ as:

$$
\frac{\partial \eta_{\bar{S}}}{\frac{\partial P(\tau)}{\partial(\tau)}}=\frac{\partial}{\frac{\partial P(\tau)}{\partial(\tau)}}\left[1+\frac{\partial P(\tau)}{\partial P(\tau)} \frac{\tau}{1-P(\tau)}\right]=\frac{\tau}{1-P(\tau)}>0
$$

This increase in $\eta_{\bar{S}}$ will lead the firm to increase its $\tau$ to reach maximum profits. 
ii. Intercept-shift in $\mathrm{P}(\tau)$

We look here at the change in FOC resulting from an intercept-shift increase in retention probabilities. That is:

$$
P_{1}(\tau)=P(\tau)+P_{0} \frac{\partial P_{1}(\tau)}{\partial \tau}=\frac{\partial P(\tau)}{\partial \tau} .
$$

Since $R(\tau)$ and $D$ are both independent from $P(\tau)$, we have $\frac{\partial \eta_{R}}{\partial P_{0}}=0$ and $\frac{\partial \eta_{D}}{\partial P_{0}}=0$. Further:

$$
\begin{aligned}
\frac{\partial \eta_{\bar{S}}}{\partial P_{0}} & =\frac{\partial}{\partial P_{0}}\left[1+\frac{\partial P_{1}(\tau)}{\partial P(\tau)} \frac{\tau}{1-P_{1}(\tau)}\right] \\
& =\frac{\partial P(\tau) \tau}{\partial(\tau)} \frac{\partial}{\partial p_{0}}\left[\frac{1}{1-P(\tau)-P_{0}}\right] \\
& =\frac{\tau}{\left(1-P(\tau)-P_{0}\right)^{2} \partial \tau} .
\end{aligned}
$$

Hence, $\partial \eta_{\bar{S}} / \partial p_{0}$ has the same sign as $\partial P(\tau) / \partial \tau$. For small $\tau$, where $\partial P(\tau) / \partial \tau$ is positive, the intercept-shift will have a positive impact on $\eta_{\bar{S}}$. For large $\tau$, where $\partial P(\tau) / \partial \tau$ is negative, the impact will be negative.

For $G M$ we have:

$$
\frac{\partial G M}{\partial p_{0}}=\frac{\partial}{\partial p_{0}} \frac{R(\tau) \cdot \bar{S}(\tau)-F C}{R(\tau) \cdot \bar{S}(\tau)}=-\frac{\partial}{\partial p_{0}} \frac{F C}{R(\tau) \cdot \bar{S}(\tau)}=\underbrace{\frac{F C}{R(\tau) \bar{S}(\tau)^{2}}}_{>0} \frac{\partial \bar{S}(\tau)}{\partial p_{0}} . \quad(\mathrm{B}-5)
$$

And:

$$
\frac{\partial \bar{S}(\tau)}{\partial p_{0}}=\frac{\partial}{\partial p_{0}} \frac{\tau . g}{1-P(\tau)-P_{0}}=\frac{\tau . g}{\left(1-P(\tau)-P_{0}\right)^{2}}>0 .
$$

Hence, an intercept-shift increase in $P(\tau)$ leads to an increase in $G M$ that leads to a decrease in FOC. Putting (B-4), (B-5), and (B-6) back into the FOC, we have that an intercept-shift increase in $P(\tau)$ will lead to higher $\tau^{*}$ if:

$$
\begin{aligned}
& \frac{\tau}{\left(1-P(\tau)-P_{o}\right)^{2}} \frac{\partial P(\tau)}{\partial \tau}>\left|\eta_{D}\right| \frac{\tau \cdot g}{\left(1-P(\tau)-P_{o}\right)^{2}} \frac{F C}{R(\tau) \bar{S}(\tau)^{2}} \\
& \frac{\partial P(\tau)}{\partial \tau}>\left|\eta_{D}\right| \frac{g}{\bar{S}(\tau)} \frac{F C}{R(\tau) \cdot \bar{S}(\tau)} \frac{\partial P(\tau)}{\partial \tau} \frac{\tau}{1-P}>\left|\eta_{D}\right| \frac{F C}{R(\tau) \cdot \bar{S}(\tau)} \eta_{\bar{S}}>\left|\eta_{D}\right| \frac{F C}{R(\tau) \cdot \bar{S}(\tau)}+1
\end{aligned}
$$

That is, if we assume that $P(\tau)$ is inverted-U shape, $\tau^{*}$ increases for small $\tau^{*}$ when there is an intercept-shift in $\mathrm{P}(\tau)$, and decreases for large $\tau^{*}$.

B. 3 change in revenue per contact

i. Change in $\partial A(\tau) / \partial \tau$ 
The revenue sensitivity affects the FOC through both $\eta_{R}$ and $\eta_{S}$. We have:

$$
\frac{\partial \eta_{R}}{\partial \frac{\partial A(\tau)}{\partial \tau}}=\frac{\partial}{\frac{\partial A(\tau)}{\partial \tau}} \frac{\partial A(\tau)}{\partial \tau} \frac{\tau}{A(\tau)-V C}=\frac{\tau}{A(\tau)-V C}>0
$$

and

$$
\begin{aligned}
\frac{\partial \eta_{\bar{S}}}{\partial \frac{\partial A(\tau)}{\partial \tau}} & =\frac{\partial}{\partial \frac{A(\tau)}{\partial \tau}}\left[1+\frac{\partial P(\tau)}{\partial \tau} \frac{\tau}{1-P(\tau)}\right] \\
& =\frac{\partial}{\partial \frac{A(\tau)}{\partial \tau}}\left[1+\frac{\tau}{1-P(\tau)} \frac{\partial}{\partial \tau} f\left(\frac{A(\tau)-c}{\tau}\right)\right] \\
& =\frac{\partial}{\partial \frac{A(\tau)}{\partial \tau}}\left[1+\frac{\tau}{1-P(\tau)} \frac{\partial f(x)}{\partial x} \frac{\partial}{\partial \tau}\left(\frac{A(\tau)-c}{\tau}\right)\right] \\
& =\frac{\partial}{\partial \frac{A(\tau)}{\partial \tau}}\left[1+\frac{\tau}{1-P(\tau)} \frac{\partial f(x)}{\partial x}\left(\frac{\frac{\partial A(\tau)}{\partial \tau}}{\tau}-\frac{A(\tau)-c}{\tau^{2}}\right)\right] \\
& =\frac{1}{1-P(\tau)} \frac{\partial f(x)}{\partial x}>0
\end{aligned}
$$

Hence, the database sensitivity to $\tau$ increases when the sensitivity of the revenue increases, creating a compounding effect that leads the firm to increase its optimal sending rate $\left(\tau^{*}\right)$.

iii. Intercept-shift in $A(\tau)$

An intercept-shift in $A(\tau)$ will be felt through $\eta_{\mathrm{R}}, \eta_{\mathrm{R}}$, and $G M$. Thus, we compute the following:

$$
\begin{gathered}
A_{1}(\tau)=A(\tau)+a_{0} \\
\frac{\partial A_{1}(\tau)}{\partial \tau}=\frac{\partial A(\tau)}{\partial \tau} \\
\frac{\partial \eta_{R}}{\partial a_{0}}=\frac{\partial}{\partial a_{0}} \frac{\partial A(\tau)}{\partial \tau} \frac{\tau}{A(\tau)-V C} \\
=\frac{\partial A(\tau)}{\partial \tau} \frac{\tau}{(A(\tau)-V C)^{2}}=\frac{-\eta_{R}}{R(\tau)}<0 \\
\frac{\partial P(\tau)}{\partial a_{0}}=\frac{\partial}{\partial a_{0}} f\left(\frac{A(\tau)-c}{\tau}\right) \\
=\frac{1}{\tau} \frac{\partial f(x)}{\partial x}>0 \\
\frac{\partial \frac{\partial P(\tau)}{\partial a_{0}}}{\partial a_{0}}=\frac{\partial}{\partial a_{0}}\left[\frac{\partial f(x)}{\partial x}\left(\frac{\partial A(\tau)}{\partial \tau} \frac{1}{\tau}-\frac{A(\tau)-c}{\tau^{2}}\right)\right] \\
=-\frac{1}{\tau^{2}} \frac{\partial f(x)}{\partial x}<0
\end{gathered}
$$




$$
\begin{aligned}
& \frac{\partial \bar{S}}{\partial a_{0}}=\frac{\partial}{\partial a_{0}}\left[\frac{\tau \cdot g}{1-P(\tau)}\right]=\tau \cdot g \frac{\frac{\partial P(\tau)}{\partial a_{0}}}{(1-P(\tau))^{2}}=\frac{\bar{S}}{1-P(\tau)} \frac{\partial P(\tau)}{\partial a_{0}}=\frac{\bar{S}}{1-P(\tau)} \frac{1}{\tau} \frac{\partial f(x)}{\partial x}>0 \\
& \frac{\partial G M}{\partial a_{0}}=-\frac{\partial}{\partial a_{0}} \frac{F C}{R(\tau) \cdot \bar{S}(\tau)} \\
& =F C\left(\frac{\frac{\partial R(\tau)}{\partial a_{0}} \cdot \bar{S}(\tau)+R(\tau) \cdot \frac{\partial \bar{S}(\tau)}{\partial a_{0}}}{(R(\tau) \cdot \bar{S}(\tau))^{2}}\right) \\
& =F C\left(\frac{\bar{S}(\tau)+\frac{R(\tau) \cdot \bar{S}(\tau)}{1-P(\tau)} \frac{1}{\tau} \frac{\partial f(x)}{\partial x}}{(R(\tau) \cdot \bar{S}(\tau))^{2}}\right) \\
& =\frac{F C}{(R(\tau) \cdot \bar{S}(\tau))}\left(\frac{1}{R(\tau)}+\frac{1}{1-P(\tau)} \frac{1}{\tau} \frac{\partial f(x)}{\partial x}\right)>0 \\
& \frac{\partial \eta_{\bar{S}}}{\partial \eta_{0}}=\frac{\partial}{\partial a_{0}}\left[1+\frac{\partial P(\tau)}{\partial \tau} \frac{\tau}{1-P(\tau)}\right] \\
& =\frac{\tau}{1-P(\tau)} \frac{\partial \frac{\partial P(\tau)}{\partial \tau}}{\partial a_{0}}+\tau \frac{\partial P(\tau)}{\partial \tau} \frac{\frac{\partial P(\tau)}{\partial a_{0}}}{\left(1-P(\tau)^{2}\right)} \\
& =\frac{\tau}{1-P(\tau)} \frac{-1}{\tau^{2}} \frac{\partial f(x)}{\partial x}+\tau \frac{\partial P(\tau)}{\partial \tau} \frac{\frac{-1}{\tau^{2}} \frac{\partial f(x)}{\partial x}}{\left(1-P(\tau)^{2}\right)} \\
& =\frac{-1}{\tau(1-P(\tau))^{2}} \frac{\partial f(x)}{\partial x}\left[(1-P(\tau))+\frac{\partial P(\tau)}{\partial \tau}\right] .
\end{aligned}
$$

Hence, $\partial \eta_{\bar{S}} / \partial a_{0}$ is negative for small to moderate levels of $\tau^{*}$ (i.e., $\left.\tau^{*}: 1-P\left(\tau^{*}\right)>-\partial P\left(\tau^{*}\right) / \partial \tau\right)$ and positive for larger $\tau^{*}$. Thus, for small to moderate $\tau^{*}$, the negative impacts on $\eta_{R}, \eta_{\bar{S}}$ and the positive impact on $G M$ will both yield a smaller $\tau^{*}$. For large $\tau^{*}$, the net impact might be positive. The point at which the effect reverses itself is given by:

$$
\begin{aligned}
& \tau: \frac{-\eta_{R}}{R(\tau)}+\frac{-1}{\tau\left(1-P(\tau)^{2}\right)}\left(\frac{\partial P(\tau)}{\partial \tau}-\tau(1-P(\tau))\right) \frac{\partial f(x)}{\partial x}+\eta_{D} \frac{F C}{R(\tau) \cdot \bar{S}(\tau)} \\
& \quad \times\left(\frac{1}{R(\tau)}+\frac{1}{1-P(\tau)} \frac{1}{\tau} \frac{\partial f(x)}{\partial x}\right)=0 .
\end{aligned}
$$

This expression is not tractable, but a lower-bound on $\tau^{*}$ is given by:

$$
\tau^{*}: \frac{\partial P(\tau)}{\partial \tau}=\tau(1-P(\tau))
$$

or $\tau^{*}: \eta_{S}=\tau^{2}+1$. 


\section{Appendix C. optimal acquisition policy}

Let us assume that the firm currently acquires $g$ customers per period for a cost of $A Q(g)$ and is considering acquiring one more. This will cost of $\triangle A Q=A Q(g+1)-$ $A Q(g)$. Between communications it will acquire $\tau g$ customer at a cost of $\tau \Delta A Q$. This is worth doing if the net present value $(N V P)$ of the additional expenses is lower than the $N P V$ of the additional revenues. The $N P V$ of additional expenses is equal to $\tau \Delta A Q \frac{e^{r \tau}}{e^{r \tau}-1}$.

Ignoring any impact of $g$ on $\tau^{*}$, the increase in revenue comes from the increase in database size: $\Delta g=1 \Rightarrow \Delta \bar{S}=\frac{\tau}{1-P}$. However, we do not go from $\bar{S}(g)$ to $\bar{S}(g+1)$ in one period, it only builds over time. The change in database size can be written as $S_{i}=\tau \sum_{j=0}^{i} p i$, with $\Delta S_{\infty}=\Delta \bar{S}$.

The change in database size affects only the revenues, it does not affect the fixed cost. Thus, we can write the change in revenue as $\Delta C E=\sum_{i=0}^{\infty} \frac{R \Delta S_{i}}{e^{i r \tau}}=R \tau \sum_{i=0}^{\infty} \frac{\sum_{j=0}^{i} P^{j} \text {. }}{e^{i r \tau}}$.
If we write the long form of the sums, we have:

$$
\begin{aligned}
\sum_{i=0}^{\infty} \frac{\sum_{j=0}^{i} P^{j}}{e^{i r \tau}}=1+\frac{(1+P)}{e^{r \tau}}+\frac{\left(1+P+P^{2}\right)}{e^{2 r \tau}} \frac{\left(1+P+P^{2}+P^{3}\right)}{e^{3^{r \tau}}}+\ldots \\
=1+\frac{P}{e^{r \tau}}+\frac{P^{2}}{e^{2 r \tau}} \frac{P^{3}}{e^{3 r \tau}}+\ldots+\frac{1}{e^{r \tau}}\left(1+\frac{P}{e^{r \tau}}+\frac{P^{2}}{e^{2 r \tau}} \frac{P^{3}}{e^{3 r \tau}}+\ldots\right) \\
+ \\
+\frac{1}{e^{2 r \tau}}\left(1+\frac{P}{e^{r \tau}}+\frac{P^{2}}{e^{2 r \tau}} \frac{P^{3}}{e^{3 r \tau}}+\ldots\right)+\frac{1}{e^{r \tau}}\left(1+\frac{P}{e^{r \tau}}+\frac{P^{2}}{e^{2 r \tau}} \frac{P^{3}}{e^{3 r \tau}}+\ldots\right)+\ldots
\end{aligned}
$$

If we substitute $D$ for the series $1+\frac{P}{e^{r \tau}}+\frac{P^{2}}{e^{2 r \tau}}+\frac{P^{3}}{e^{3 r \tau}}+\cdots=\frac{e^{r \tau}}{e^{r \tau}-P}$, then we can re-write the sums as:

$$
\sum_{i=0}^{\infty} \frac{\sum_{j=0}^{i} P^{j}}{e^{i r \tau}}=D+\frac{D}{e^{a r \tau}}+\frac{D}{e^{a r \tau}} \quad=D \frac{e^{r \tau}}{e^{r \tau}-1}
$$

Thus, $\Delta C E=R \tau \sum_{i=0}^{\infty} \frac{\sum_{j=0}^{i} P^{j}}{e^{i r \tau}}=R \tau \frac{e^{r \tau}}{e^{r \tau}-P} \frac{e^{r \tau}}{e^{r \tau}-1}$ and:

$$
\triangle C E>N P V(\triangle A Q)
$$

$$
\Leftrightarrow R \tau \frac{e^{r \tau}}{e^{r \tau}-P} \frac{e^{r \tau}}{e^{r \tau}-1}>\tau \Delta A Q \frac{e^{r \tau}}{e^{r \tau}-1} \Leftrightarrow R \frac{e^{r \tau}}{e^{r \tau}-P}>\Delta A Q \Leftrightarrow C L V>\Delta A Q
$$

In other words, the optimal acquisition policy from a profit perspective is to spend up to the $C L V$ on acquisition.

\section{References}

Bayon, T., Gutsche, J., \& Bauer, H. (2002). Customer equity marketing: touching the intangible. European Management Journal, 20(3), 213-22.

Berger, P. D., Bolton, R. N., Bowman, D., Elten Briggs, V., Kumar, A. P., \& Terry, C. (2002). Marketing actions and the value of customer assets: a framework for customer asset management. Journal of Service Research, 5(1), 39-54. 
Berger, P. D., \& Nasr, N. I. (1998). Customer life time value: marketing models and applications. Journal of Interactive Marketing, 12(1), 17-30.

Blattberg, Robert C. and John Deighton (1996), "Manage Marketing by the Customer Equity Test," Harvard Business Review, July-August, 136-144.

Fader, P. S., Hardie, B. G. S., \& Lee, K. L. (2005). RFM and CLV: Using iso-value curves for customer base analysis. Journal of Marketing Research, XLII, 415-30.

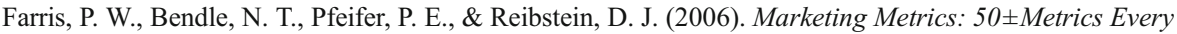
Executive Should Master. New Jersey: Wharton School Publishing.

Fruchter, G. E., \& Zhang, J. Z. (2004). Dynamic targeted promotions: a customer retention and acquisition perspective. Journal of Service Research, 7(1), 3-19.

Gupta, Sunil, Lehmann, D. R., \& Stuart, J. A. (2004). Valuing customers. Journal of Marketing Research, XLI, 7-18.

Haenlein, Michael, Kaplan, A. M., \& Detlef, Schoder. (2006). Valuing the real option of abandoning unprofitable customers when calculating customer lifetime value. Journal of Marketing, 70, 5-20.

Hwang, H., Jung, T., \& Suh, E. (2004). An LTV model and customer segmentation based on customer value: a cose study on the wireless telecommunication industry. Expert Systems with Applications, 26 (2004), 181-8.

Libai, B., Narayandas, D., \& Humby, C. (2002). Toward an Individual Customer Profitability Model: A Segment-Based Approach. Journal of Service Research, 5(1), 69-76.

Little, J. D. C. (1961). A proof of the queuing formula: $L=\lambda W$. Operations Research, 9, 383-7.

Reinartz, W. J., Thomas, J. S., \& Kumar, V. (2005). Balancing acquisition and retention resources to maximize customer profitability. Journal of Marketing, 69, 63-79.

Reinartz, W. J., \& Kumar, V. (2003). The impact of customer relationship characteristics on profitable lifetime duration. Journal of Marketing, 67, 77-99.

Rust, R. T., Lemon, K. N., \& Zeithaml, V. A. (2004). Return on marketing: using customer equity to focus marketing strategy. Journal of Marketing, 68, 109-27.

Sweeny, James L. (1992), "Economic Theory of Depletable Resources: An Introduction,” in Handbook of Natural Resources and Energy Economics, Vol. 3, ed. by A. V. Kneese and J. L. Sweeney, NorthHolland.

Venkatesan, R., \& Kumar, V. (2004). A customer lifetime value framework for customer selection and resource allocation strategy. Journal of Marketing, 68, 106-25.

Villanueva, J., Yoo, S., \& Hanssens, D. M. (2008). The impact of marketing-induced versus word-ofmouth customer acquisition on customer equity growth. Journal of Marketing Research, XLV, 48-59.

Wang, P., \& Spiegel, T. (1994). Database marketing and its measurement of success: designing a managerial instrument to calculate the value of a repeat customer database. Journal of Direct Marketing, 8(2), 73-81.

Winer, R. S. (2001). A framework for customer relationship management. California Management Review, 43, 89-105.

Zeithaml, V. A., Rust, R. T., \& Lemon, K. N. (2001). The customer pyramid: creating and serving profitable customers. California Management Review, 43(4), 118-42. 